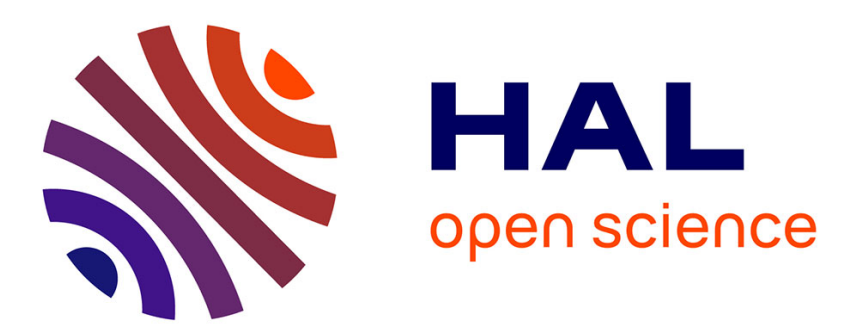

\title{
Mathematical properties of a class of integro-differential models from population genetics *
}

\author{
M-E Gil, F Hamel, Guillaume Martin, L Roques
}

\section{To cite this version:}

M-E Gil, F Hamel, Guillaume Martin, L Roques. Mathematical properties of a class of integrodifferential models from population genetics *. SIAM Journal on Applied Mathematics, 2017, 77 (4), pp.1536-1561. 10.1137/16M1108224 . hal-01406163

\section{HAL Id: hal-01406163 \\ https://hal.science/hal-01406163}

Submitted on 30 Nov 2016

HAL is a multi-disciplinary open access archive for the deposit and dissemination of scientific research documents, whether they are published or not. The documents may come from teaching and research institutions in France or abroad, or from public or private research centers.
L'archive ouverte pluridisciplinaire HAL, est destinée au dépôt et à la diffusion de documents scientifiques de niveau recherche, publiés ou non, émanant des établissements d'enseignement et de recherche français ou étrangers, des laboratoires publics ou privés. 


\title{
Mathematical properties of a class of integro-differential models from population genetics*
}

\author{
M.-E. Gil ${ }^{\mathrm{a}, \mathrm{b}}$, F. Hamel ${ }^{\mathrm{a}}$, G. Martin ${ }^{\mathrm{c}}$ and L. Roques ${ }^{\mathrm{b}}$ \\ a Aix Marseille Univ, CNRS, Centrale Marseille, I2M, Marseille, France \\ b BioSP, INRA, 84914, Avignon, France \\ c ISEM (UMR 5554), CNRS, 34095, Montpellier, France
}

November 22, 2016

\begin{abstract}
We propose a mathematical analysis of an integro-differential model arising in population genetics. The model describes the dynamics of fitness distribution in an asexual population under the effect of mutation and selection. These two processes are represented by two nonlocal terms. First, we prove the existence and uniqueness of the solution, and we derive asymptotic estimates of the distribution as the fitness tends to $\pm \infty$. Based on these asymptotic estimates, we show that the cumulant generating function of the distribution is well-defined and satisfies a linear nonlocal transport equation that we solve explicitly. This explicit formula allows us to characterize the dependence of the long time behavior of the distribution with respect to the mutation kernel. On the one hand, if the kernel contains some beneficial mutations, the distribution diverges, which is reminiscent of the results of Alfaro and Carles (2014) who analysed a mutator-replicator equation with a diffusive mutation term. On the other hand, if the initial fitness distribution admits some upper bound, purely deleterious kernels lead to the convergence of the distribution towards an equilibrium. The shape of the equilibrium distribution strongly depends on the kernel through its harmonic mean $-s_{H}$ : the distribution admits a positive mass at the best initial fitness class if and only if $s_{H} \neq 0$.
\end{abstract}

\section{Introduction}

The study of the dynamics of fitness distributions in asexual populations is a fundamental issue in population genetics. It has implications for the evolution of microbial pathogens (many of

*This work has been carried out in the framework of Archimède Labex (ANR-11-LABX-0033) and of the A*MIDEX project (ANR-11-IDEX-0001-02), funded by the "Investissements d'Avenir" French Government programme managed by the French National Research Agency (ANR). The research leading to these results has also received funding from the ANR within the projects NONLOCAL ANR-14-CE25-0013 and MECC, ANR-13-ADAP-0006 and from the European Research Council under the European Union's Seventh Framework Programme (FP/2007-2013) / ERC Grant Agreement n.321186 - ReaDi - Reaction-Diffusion Equations, Propagation and Modelling. 
which mostly reproduce asexually), cancer cells or invasive clonal plants and animals. It is also important for the evolution of microbial populations in controlled environments (e.g. in bioreactors) and can in principle be compared to an increasing body of empirical data generated by experimental evolution (reviewed in Elena and Lenski, 2003).

In several recent studies (Gerrish et al., 2007; Sniegowski and Gerrish, 2010; Desai and Fisher, 2011), the distribution of the Malthusian fitness $m$ in a population was described as the solution $p(t, m)$ of the integro-differential equation

$$
\partial_{t} p(t, m)=U(J \star p-p)(t, m)+p(t, m)(m-\bar{m}(t)), t \geq 0, m \in \mathbb{R},
$$

where

$$
\left\{\begin{aligned}
(J \star p-p)(t, m) & =\int_{\mathbb{R}} J(m-y) p(t, y) d y-p(t, m)=\int_{\mathbb{R}} J(m-y)(p(t, y)-p(t, m)) d y, \\
\bar{m}(t) & =\int_{\mathbb{R}} m p(t, m) d m .
\end{aligned}\right.
$$

Here, the coefficient $U>0$ is the mutation rate per unit time (assumed constant across individuals and time), $J$ is denoted the mutation kernel and $\bar{m}(t)$ the mean fitness in the population at time $t$. The first term $U(J \star p-p)(t, m)$ and the second term $p(t, m)(m-\bar{m}(t))$ in the right hand side of (1) respectively describe the effects of mutation and selection on the dynamics of fitness distributions.

This equation naturally arises as an extension of the classical selection equation $\partial_{t} p(t, m)=$ $p(t, m)(m-\bar{m}(t))$ (Tsimring et al., 1996) to include arbitrary non-epistatic mutation effects: $m \mapsto J(m-y)$ is the probability density function describing the fitness of a mutant offspring, given that its parent has fitness $y$. In studies dealing with finite population sizes, the dynamics of fitness distributions are often described by stochastic "Langevin" integro-differential equations, corresponding to (1) with an additional noise term in the right-hand side to account for the effect of genetic drift (Desai and Fisher, 2011; Good et al., 2012; Good and Desai, 2013, 2014).

To our knowledge, all of the studies which rely on the deterministic equation (1) or its stochastic counterpart, although eminently informative and useful in the field of population genetics, are based on formal computations rather than rigorous mathematical arguments. The mathematical study of (1) combines at least three difficulties, compared to standard reaction-diffusion equations $\partial_{t} u=D \partial_{x x} u+f(u)$ with local diffusion and local reaction term :

- the mutation term $U(J \star p-p)(t, m)$ is nonlocal and has no regularizing properties. This term appears in reaction-dispersion equations with nonlocal dispersion terms, of the form $\partial_{t} u=(J \star u-u)+f(u)$, which have been extensively studied, but only for local reaction terms $f(u(t, m))$. Most of the literature on these equations focus on the existence/nonexistence of traveling wave solutions and other spreading properties (Alfaro and Coville, 2016; Carr and Chmaj, 2004; Coville and Dupaigne, 2007; Garnier, 2011; Garnier et al., 2016; Schumacher, 1980; Weinberger, 1982, 2002; Yagisita, 2009);

- the selection term $p(t, m)(m-\bar{m}(t))$ is also nonlocal (and nonlinear) due to the term $\bar{m}(t)$. Reaction-diffusion equations with nonlocal reaction terms of the type $f(u)=$ $u(1-\phi \star u)$ (with $\phi \geq 0$ of integral 1) have been investigated in the recent works of e.g. Berestycki et al. (2009), Fang and Zhao (2011), Faye and Holzer (2015), Genieys et al. (2006), Gourley (2000) and Hamel and Ryzhik (2014); see also the work of Alfaro et al. 
(2014) for reaction terms of the form $f(u)=u(u-\theta)(1-\phi \star u)$, where $\theta$ is a positive constant. One of the main difficulties in the study of these equations was the lack of maximum principle or comparison principle;

- the selection term $p(t, m)(m-\bar{m}(t))$ has an unbounded coefficient $(m)$, which may lead to finite time blow-up (Alfaro and Carles, 2014).

By replacing the mutation term $U(J \star p-p)(t, m)$ with a Laplace operator $\partial_{m m} p$, Alfaro and Carles (2014) have derived rigorous mathematical results on the so-called replicator-mutator equation

$$
\partial_{t} p(t, m)=\partial_{m m} p(t, m)+p(t, m)(m-\bar{m}(t)), t>0, m \in \mathbb{R} .
$$

In particular, they derived an exact expression for the solution $p(t, m)$ of the Cauchy problem associated with (3) and they showed that this solution is well-defined (and unique) up to a time $T$. This time $T$ is infinite if $p_{0}$ decays faster than any exponential, it is finite if $p_{0}(m)=p(0, m)$ decays exponentially as $m \rightarrow+\infty$ (blow-up in finite time), while it is equal to 0 if $p_{0}$ is fat-tailed (i.e., decays slower than any exponential, this last situation corresponds to instantaneous blow-up). In all cases, the model becomes unrealistic after some time, even if $T=+\infty$ : namely, $\bar{m}(t)$ behaves like $t^{2}$ as $t \rightarrow+\infty$, whereas experimental results suggest that $\bar{m}(t)$ should increase at most linearly (see e.g. Tsimring et al., 1996). A similar drawback is expected for the model (1) if the kernel $J$ accounts for beneficial mutations, i.e., if the support of $J, \operatorname{supp}(J)$, satisfies $\operatorname{supp}(J) \cap(0,+\infty) \neq \emptyset$. On the other hand, purely deleterious kernels which satisfy $\operatorname{supp}(J) \cap(0,+\infty)=\emptyset$ should lead to more realistic results, qualitatively very different from those obtained with the diffusion approximation (3). These kernels arise when only deleterious mutations contribute to the evolutionary dynamics, as is expected for example in a stable environment with a well adapted population.

The aim of this work is to set a firm mathematical basis for the main properties of the solution of (1). In particular, we derive sufficient conditions (which we conjecture to be optimal in some sense) for the existence and uniqueness of the solution of the Cauchy problem associated with (1). We also give upper bounds for the asymptotic behaviour of the solution as $|m| \rightarrow+\infty$, which enables us to define the cumulant generating function of the fitness distribution:

$$
C(t, z)=\ln \left(\int_{\mathbb{R}} p(t, m) \mathrm{e}^{z m} d m\right), t \geq 0, z \geq 0,
$$

and to derive an explicit formula for $C$ as the solution of a nonlocal linear transport equation which had been only formally derived in (Martin and Roques, 2016). Based on this formula, we infer explicit expressions for the first centered moments $\bar{m}(t)$ and $V(t)$ of the distribution $p$ and we analyze the large time behaviour of $p$, which we find to be strongly dependent on the type of mutation kernel. Our main assumptions and results are described in the next section.

\section{Assumptions and main results}

As mentioned above for the diffusion approximation (3), Alfaro and Carles (2014) have shown that global existence (in time) of the solution of the Cauchy problem is possible only if the initial condition $p_{0}(m)=p(0, m)$ decays faster than any exponential function, in the sense that the quantity

$$
\Lambda=\sup \left\{\alpha \geq 0, \quad \int_{0}^{+\infty} \mathrm{e}^{\alpha m} p_{0}(m) d m<+\infty\right\}
$$


in (Alfaro and Carles, 2014, Theorem 2.3) is infinite. In this paper, a similar assumption is required to get global existence. More precisely, we assume throughout this paper that the initial distribution of fitness $p_{0} \in L^{\infty}(\mathbb{R})$ is a probability density function, that is,

$$
p_{0} \geq 0 \text { a.e. in } \mathbb{R} \text { and } \int_{\mathbb{R}} p_{0}(m) d m=1 .
$$

Additionally, we assume that $p_{0}$ satisfies

$$
\lim _{m \rightarrow \pm \infty} p_{0}(m) \mathrm{e}^{\alpha|m|}=0 \text { for all } \alpha>0 .
$$

Regarding the mutation kernel $J$, we assume that $J \in L^{1}(\mathbb{R})$ with

$$
\int_{\mathbb{R}} J(m) d m=1 \text { and } J(m) \geq 0 \text { for almost every (a.e.) } m \in \mathbb{R} \text {. }
$$

Furthermore, the proof of global existence in time is based on the assumption that $J(m)$ decays faster than any exponential function as $|m| \rightarrow+\infty$, in the sense that

$$
\int_{\mathbb{R}} J(m) \mathrm{e}^{\alpha|m|} d m<+\infty \text { for all } \alpha>0
$$

Remark 2.1 If $J$ were assumed to be just a measure of the type

$$
J=\rho \delta_{0}+(1-\rho) \widetilde{J}
$$

for some $\rho \in[0,1]$ and a function $\widetilde{J} \in L^{1}(\mathbb{R})$ satisfying (6), where $\delta_{0}$ denotes the Dirac measure at 0 , then (1) would be equivalent to the following equation:

$$
\partial_{t} p(t, m)=U(1-\rho)(\widetilde{J} \star p-p)(t, m)+p(t, m)(m-\bar{m}(t)), t>0, m \in \mathbb{R} .
$$

As expected intuitively, having a kernel $J$ of the type (8) with a non-zero mass at 0 is equivalent to considering a lower mutation rate $U(1-\rho)$ with the mutation kernel $\widetilde{J}$ (i.e. considering only the mutation rate and effects to non-neutral mutations).

\subsection{Existence, uniqueness and properties of the solution}

We are now in position to state our existence and uniqueness result.

Theorem 2.2 (Existence, uniqueness, exponential decay) Assume that $p_{0} \in L^{\infty}(\mathbb{R})$ satisfies assumptions (4)-(5) and $J \in L^{1}(\mathbb{R})$ satisfies assumptions (6)-(7). Then problem (1) with initial condition $p_{0}$ admits a unique solution $p \geq 0$ such that

(i) $p \in \mathcal{C}^{1}\left([0,+\infty), L^{\infty}(\mathbb{R})\right), \bar{m} \in \mathcal{C}([0,+\infty))$ and

$$
\int_{\mathbb{R}} p(t, m) d m=1 \text { for all } t \geq 0 ;
$$


(ii) $p$ decays faster than any exponential function as $|m| \rightarrow+\infty$ in the sense that, for every $\alpha>0$ and $T>0$, there is $\Gamma_{\alpha, T}>0$ such that:

$$
0 \leq p(t, m) \leq \Gamma_{\alpha, T} \mathrm{e}^{-\alpha|m|} \text { for all } t \in[0, T] \text { and a.e. } m \in \mathbb{R}^{1}
$$

Lastly, the same decay property (9) holds good for $\left|\partial_{t} p(t, m)\right|$ as well.

Remark 2.3 The assumptions (5) and (7) on the decay of $p_{0}$ and $J$ can be slightly relaxed: if one of these functions (or both) decays only exponentially fast, i.e., if $p_{0}$ or $J$ satisfy (5) and (7) for a given $\alpha>0$, then local existence (that is, existence on a finite interval $[0, T]$ ) remains true. This follows from the proof of Proposition 4.1 below. We conjecture however that the assumptions (5) and (7) are somehow optimal to get global existence in time. In particular, "fat-tailed" kernels at $m=+\infty$ (i.e., $J(m) \mathrm{e}^{\alpha m} \rightarrow+\infty$ as $m \rightarrow+\infty$ for every $\alpha>0$ ) such as those appearing in the reaction-dispersion equations of (Garnier, 2011; Garnier et al., 2016) should lead to instantaneous blow-up at $t=0$. This conjecture is based on the following heuristic argument: the solution of the equation $\partial_{t} p=(J \star p-p)$ (without the selection term) decays slower than any exponential at $m=+\infty$, at any time $t>0$, even if the initial condition is compactly supported: this follows from the existence of a subsolution of the type $\left(p_{0}(m)+t\left(J \star p_{0}\right)(m)\right) e^{-t}$ constructed in (Garnier, 2011). Thus, the situation is similar to the case of a fat-tailed initial condition, which is known to lead to instantaneous blow-up in the diffusion case (Alfaro and Carles, 2014), due to the interplay with the unbounded coefficient $m$ in $p(t, m)(m-\bar{m}(t))$.

If the kernel $J$ corresponds to purely deleterious mutations, i.e. $\operatorname{supp}(J) \subset(-\infty, 0]$, the expression (9) can be made more precise. In such a case, it is natural to expect that the upper bound of the support $\operatorname{supp}(p(t, \cdot))$ of $p(t, \cdot)$ remains lower or equal to that of $p_{0}$. This is the purpose of the following proposition.

Proposition 2.4 (Purely deleterious kernels) We assume that $p_{0} \in L^{\infty}(\mathbb{R})$ satisfies the assumptions (4)-(5) and that the mutation kernel $J \in L^{1}(\mathbb{R})$ satisfies the assumptions (6)-(7) and is purely deleterious, that is,

$$
J(m)=0 \text { for a.e. } m \geq 0 .
$$

Let

$$
m_{0}=\sup \left(\operatorname{supp}\left(p_{0}\right)\right)=\sup \left\{m \in \mathbb{R} \text { s.t. } \operatorname{supp}\left(p_{0}\right) \not \subset(-\infty, m)\right\} \in(-\infty,+\infty] .
$$

Then, the solution $p$ of (1) given in Theorem 2.2 with initial condition $p_{0}$ is such that

$$
\operatorname{supp}(p(t, \cdot)) \subset\left(-\infty, m_{0}\right] \text { for all } t \geq 0 .
$$

\footnotetext{
${ }^{1}$ Throughout the paper, with a slight abuse of notation, we say that a function $u: I \times \mathbb{R} \rightarrow \mathbb{R},(t, m) \mapsto$ $u(t, m)$ belongs to $\mathcal{C}^{k}(I, W)$ for some integer $k$, some interval $I \subset \mathbb{R}$ and some normed space $W$, if $t \mapsto u(t, \cdot)$ maps $I$ to $W$ and is of class $\mathcal{C}^{k}(I, W)$. In particular, here, the fact that $p \in \mathcal{C}^{1}\left([0,+\infty), L^{\infty}(\mathbb{R})\right)$ solves $(1)$ implies that, for every $t \geq 0$, (1) is satisfied for a.e. $m \in \mathbb{R}$. Furthermore, throughout the paper, when we write that an equality or an inequality holds for all $t$ in some interval $I$ and a.e. $m \in \mathbb{R}$, that means that, for every $t \in I$, it holds for a.e. $m \in \mathbb{R}$.
} 


\subsection{The cumulant generating function (CGF)}

Using the fact that the solution $p(t, m)$ of (1) decays as $|m| \rightarrow+\infty$ faster than any exponential according to Theorem 2.2, we can define, for all $t \geq 0$ and $z \geq 0,{ }^{2}$ the cumulant generating function (CGF) of the fitness distribution:

$$
C(t, z):=\ln \left(\int_{\mathbb{R}} p(t, m) \mathrm{e}^{z m} d m\right) .
$$

The CGF provides many informations on the underlying distribution. In particular, it is easily seen that

$$
\partial_{z} C(t, 0)=\int_{\mathbb{R}} m p(t, m) d m
$$

is the mean fitness $\bar{m}(t)$ at time $t$. Furthermore,

$$
\partial_{z z} C(t, 0)=\int_{\mathbb{R}} m^{2} p(t, m) d m-(\bar{m}(t))^{2}
$$

is the variance in fitness $V(t)$. More generally, all moments $\int_{\mathbb{R}} m^{k} p(t, m) d m$ of the distribution $p(t, \cdot)$ can be retrieved from the CGF $C(t, \cdot)$ at time $t$.

Let us now derive the equation satisfied by $C$ and give an explicit formula for $C$. For any given $t \geq 0$ and $z \geq 0$, by multiplying equation (1) by $\mathrm{e}^{z m}$ and by integrating over $\mathbb{R}$ with respect to $m$ (all integrals below converge due to the decay properties of $p(t, \cdot)$ and $\partial_{t} p(t, \cdot)$ given in Theorem 2.2), we obtain:

$$
\begin{aligned}
\int_{\mathbb{R}} \mathrm{e}^{z m} \partial_{t} p(t, m) d m= & U \int_{\mathbb{R}} \mathrm{e}^{z m}(J \star p-p)(t, m) d m+\int_{\mathbb{R}} m \mathrm{e}^{z m} p(t, m) d m \\
& -\bar{m}(t) \int_{\mathbb{R}} \mathrm{e}^{z m} p(t, m) d m \\
= & U\left(\int_{\mathbb{R}} J(m) \mathrm{e}^{z m} d m\right)\left(\int_{\mathbb{R}} \mathrm{e}^{z m} p(t, m) d m\right)-U \int_{\mathbb{R}} \mathrm{e}^{z m} p(t, m) d m \\
& +\int_{\mathbb{R}} m \mathrm{e}^{z m} p(t, m) d m-\bar{m}(t) \int_{\mathbb{R}} \mathrm{e}^{z m} p(t, m) d m .
\end{aligned}
$$

Dividing the last equality by $\int_{\mathbb{R}} \mathrm{e}^{z m} p(t, m) d m$, we obtain that $C$ is a classical $\mathcal{C}^{1}([0,+\infty) \times$ $[0,+\infty)$ ) (from Lebesgue's dominated convergence theorem) solution of the following non-local equation

$$
\left\{\begin{aligned}
\partial_{t} C(t, z) & =\partial_{z} C(t, z)-\partial_{z} C(t, 0)+\beta(z), & & t \geq 0, z \geq 0 \\
C(0, z) & =C_{0}(z), & & z \geq 0 \\
C(t, 0) & =0, & & t \geq 0
\end{aligned}\right.
$$

with

$$
\beta(z)=U\left(\int_{\mathbb{R}} J(m) \mathrm{e}^{z m} d m-1\right) \text { and } C_{0}(z)=\ln \left(\int_{\mathbb{R}} p_{0}(m) \mathrm{e}^{z m} d m\right) .
$$

The boundary condition $C(t, 0)=0$ directly follows from property (i) of Theorem 2.2. We also point out that, from (4)-(7), the functions $\beta$ and $C_{0}$ are actually of class $\mathcal{C}^{\infty}([0,+\infty))$. The theorem below then gives the explicit formula for $C(t, z)$.

\footnotetext{
${ }^{2}$ The quantity $C(t, z)$ given in (12) can also be defined for all $z<0$, but we only use in the sequel the properties of $C(t, z)$ for $z \geq 0$, as well as the asymptotic properties of $C(0, z)$ as $z \rightarrow+\infty$.
} 
Theorem 2.5 (Explicit formula for the CGF) The solution of problem (13) is unique and is given by

$$
C(t, z)=C_{0}(z+t)-C_{0}(t)+\int_{0}^{t}(\beta(z+s)-\beta(s)) d s, \quad t \geq 0, z \geq 0 .
$$

Using this formula, we obtain simple expressions for the mean fitness and the variance in fitness.

Corollary 2.6 For every $t \geq 0$, the mean fitness is given by

$$
\bar{m}(t)=\partial_{z} C(t, 0)=C_{0}^{\prime}(t)+\beta(t)
$$

and the variance in fitness is given by

$$
V(t)=\partial_{z z} C(t, 0)=C_{0}^{\prime \prime}(t)+\beta^{\prime}(t)-\beta^{\prime}(0) .
$$

Remark 2.7 When the mutation term $U(J \star p-p)$ is replaced with a diffusion term $\partial_{m m} p$, equation (1) becomes (3), that is,

$$
\partial_{t} p=\partial_{m m} p+(m-\bar{m}(t)) p .
$$

With the assumptions (4)-(5) on $p_{0}$ the solution $p$ of (3) satisfies property (9) (see Alfaro and Carles (2014)). In this case, the function $C$ defined by (12) satisfies (13) with $\beta(z)=z^{2}$. Thus, we can apply Theorem 2.5 and Corollary 2.6, which show that

$$
\bar{m}(t)=C_{0}^{\prime}(t)+t^{2}=\frac{\int_{\mathbb{R}} m p_{0}(m) \mathrm{e}^{t m} d m}{\int_{\mathbb{R}} p_{0}(m) \mathrm{e}^{t m} d m}+t^{2} .
$$

We therefore retrieve the results of (Alfaro and Carles, 2014, Corollary 2.2).

\subsection{Large time behaviour}

Using the explicit formulas of Theorem 2.5 and Corollary 2.6, we now describe the large time behaviour of the fitness distribution in terms of the kernel $J$. In particular we distinguish two main types of kernels: those including beneficial mutations and the purely deleterious kernels.

Theorem 2.8 (Large time behaviour) Under the assumptions of Theorem 2.2, we have:

(i) if $J$ is a purely deleterious kernel, i.e. $\operatorname{supp}(J) \subset(-\infty, 0]$, and if the quantity $m_{0}$ defined in (11) is finite, then

$$
\lim _{t \rightarrow+\infty} \bar{m}(t)=m_{0}-U<m_{0} \text { and } \lim _{t \rightarrow+\infty} V(t)=-U \mu_{J}>0
$$

where $\mu_{J}=\int_{\mathbb{R}} m J(m) d m=\int_{-\infty}^{0} m J(m) d m<0$ is the mean effect of mutations on fitness; 
(ii) if $J$ is a purely deleterious kernel and if $m_{0}=+\infty$, then

$$
\lim _{t \rightarrow+\infty} \bar{m}(t)=+\infty \quad \text { and, if } C_{0}^{\prime \prime}(+\infty) \text { exists, } \lim _{t \rightarrow+\infty} V(t)=C_{0}^{\prime \prime}(+\infty)-U \mu_{J}
$$

(iii) if $J$ includes some beneficial mutations, i.e. $\operatorname{supp}(J) \cap(0,+\infty) \neq \emptyset$, then

$$
\lim _{t \rightarrow+\infty} \bar{m}(t)=+\infty \text { and } \lim _{t \rightarrow+\infty} V(t)=+\infty .
$$

Case (i) corresponds to the classic result on mutation-selection balance with purely deleterious mutations: the mutation load (i.e., the quantity $m_{0}-\bar{m}(+\infty)$ ) is equal to the mutation rate $U$. This load can only be defined if there is an upper bound to the initial fitness distribution (a genotype of maximal fitness). Otherwise if the maximal fitness is unbounded (all else being equal) we retrieve case (ii), where the variance in fitness may or may not stabilize to a finite limit but the mean fitness increases without bounds. The former arises for example when the initial fitness distribution is Gaussian $\left(C_{0}^{\prime \prime}(z)=\sigma^{2}\right.$ for all $\left.\mathrm{z} \in \mathbb{R}^{+}\right)$: the mean fitness diverges to infinity in spite of a purely deleterious kernel while the variance converges to $\sigma^{2}-U \mu_{J}$. Finally in case (iii) the presence of beneficial mutations implies that both the mean and variance in fitness diverge to infinity over time.

In the case (i) (purely deleterious kernels and $\left.\sup \left(\operatorname{supp}\left(p_{0}\right)\right)<+\infty\right)$, we will actually show that $p(t,$.$) converges in a weak sense to a measure p_{\infty}$. In this respect, we use a standard result (see e.g. Martin-Löf, 1973) which connects convergence in CGF of a sequence of random variables with convergence in distribution.

Theorem 2.9 (Continuity theorem for CGFs, Martin-Löf (1973)) Let $(p(t, \cdot))_{t \geq 0}$ be a family of $L^{\infty}(\mathbb{R})$ probability densities on $\mathbb{R}$, in the sense of (4), and assume that the cumulant generating function $C(t, z)=\ln \left(\int_{\mathbb{R}} p(t, m) \mathrm{e}^{z m} d m\right)$ is defined on $[0,+\infty) \times[0,+\infty)$. If

$$
\lim _{t \rightarrow+\infty} C(t, z)=C_{\infty}(z) \text { for every } z \geq 0
$$

and if $C_{\infty}$ is continuous at $z=0$, then $p(t,$.$) converges weakly as t \rightarrow+\infty$ to a nonnegative measure $p_{\infty}$ and

$$
C_{\infty}(z)=\ln \left(\int_{\mathbb{R}} \mathrm{e}^{z m} d p_{\infty}(m)\right) \text { for all } z \geq 0 .
$$

By weak convergence, we mean that

$$
\lim _{t \rightarrow+\infty} \int_{\mathbb{R}} \phi(m) p(t, m) d m=\int_{\mathbb{R}} \phi(m) d p_{\infty}(m)
$$

for any bounded continuous function $\phi$.

Coming back to case (i) of Theorem 2.8 for our problem (1), it turns out that the measure $p_{\infty}$ exists and that it strongly depends on the harmonic mean $-s_{H}$ of the (purely deleterious) kernel $J$ defined by

$$
-s_{H}:=\left(\int_{\mathbb{R}} \frac{J(m)}{m} d m\right)^{-1}=\left(\int_{-\infty}^{0} \frac{J(m)}{m} d m\right)^{-1} \in(-\infty, 0] .
$$

More precisely, we will show that, in the purely deleterious case with $m_{0}<+\infty$, then $p_{\infty}$ admits a positive mass at $m_{0}$-meaning that a positive proportion $\rho$ of the population has the best possible genotype- if and only if $s_{H} \neq 0$. The next theorem summarizes these results and gives the value of $\rho$, in terms of $s_{H}$. 
Theorem 2.10 (Large time behaviour if $J$ is purely deleterious and if $m_{0}$ is finite) Under the assumptions of Theorem 2.2, if $J$ is purely deleterious, i.e. $\operatorname{supp}(J) \subset(-\infty, 0]$, and if the quantity $m_{0}$ defined in (11) is finite, then the probability densities $p(t,$.$) converge weakly$ as $t \rightarrow+\infty$ to a nonnegative measure $p_{\infty}$ with $C G F$

$$
C_{\infty}(z)=U \int_{-\infty}^{0} \frac{J(m)}{m}\left(1-\mathrm{e}^{z m}\right) d m+m_{0} z
$$

Furthermore, $p_{\infty}$ can be written as a sum of two measures:

$$
p_{\infty}=\rho \delta_{m_{0}}+(1-\rho) p^{\star},
$$

where $\rho \in[0,1], \delta_{m_{0}}$ is the Dirac measure at $m_{0}$ and $p^{\star}$ is a nonnegative measure supported in $\left(-\infty, m_{0}\right]$. Furthermore, $p^{\star}\left(\left(-\infty, m_{0}\right]\right)=1$ and $p^{\star}$ has no mass at $m_{0}$ in the sense that $p^{\star}\left(\left[m_{0}-\varepsilon, m_{0}\right]\right) \rightarrow 0$ as $\varepsilon \rightarrow 0$. Lastly,

(a) if $s_{H} \neq 0$, then the weight $\rho$ of the genotype with fitness $m_{0}$ is equal to $\rho=\mathrm{e}^{-U / s_{H}} \in(0,1)$,

(b) if $s_{H}=0$, then the weight $\rho$ of the genotype with fitness $m_{0}$ is $\rho=0$.

A tentative intuition of this result can be provided as follows. A non-zero harmonic mean $\left(s_{H} \neq 0\right)$ means that the mutation kernel $J(m)$ is in some sense small around $m=0$. This means that there are no mutants with vanishingly small deleterious effects, so that the initial fittest class (at $m=m_{0}$ ) is preserved by selection in the face of mutation. Therefore, a mass at the optimal fitness emerges in the large time behaviour of the solution. Note that this case arises with any discrete distribution of mutation effects. On the contrary, a zero harmonic mean $\left(s_{H}=0\right)$, implies that mutants with vanishingly small selection coefficients arise with increasing frequencies. Thus, many mutants produced are almost not selected against and can therefore be preserved, depleting the initial class of optimal genotypes. Ultimately, this class vanishes (being replaced by a bulk of very weakly deleterious mutants).

\section{Discussion}

We were able to provide qualitative existence and uniqueness results as well as more quantitative characterizations of the distributions solving (1), for a wide class of mutation kernels, that may include beneficial mutations or not.

We believe that the most interesting cases arise when purely deleterious kernels are considered. In such cases, numerical computations and formal analyses conduced in Martin and Roques (2016) indicate that the solution of (1) accurately describes empirical distributions obtained by stochastic individual based simulations. Additionally, if the initial fitness distribution admits some upper bound, the distribution converges towards an equilibrium. As already observed by Martin and Roques (2016), based on formal computations, the shape of the equilibrium strongly depends on the kernel through its harmonic mean $-s_{H}$. We have shown here, based on rigorous mathematical arguments, that the equilibrium admits a positive mass at the initial fittest class if and only if $s_{H} \neq 0$, i.e., if mutation does not produce too many nearly optimal genotypes. The weight of this mass is then $e^{-U / s_{H}}$ : it decays exponentially with the mutation rate $U$.

As already discussed in the Introduction, in those models which implicitly assume infinite population sizes, taking beneficial mutations into account ultimately leads to unrealistic results, 
compared to stochastic simulation models and experiments. This was already observed in the diffusion case by Alfaro and Carles (2014). This is still the case here, with a mean fitness $\bar{m}(t)$ which increases exponentially fast (see Corollary 2.6 and the proof of Theorem 2.8), whereas stochastic simulation models lead to a constant average rate of adaptation $\nu$ at large times (see Gerrish and Lenski (1998); Good et al. (2012), and Park et al. (2010) for a review). However, as discussed by Martin and Roques (2016), the approach used here seems to remain accurate even with reasonable population sizes $\left(\approx 10^{6}\right)$ over a possibly long initial period of several 100 or 1000 generations (depending on the kernel).

The deterministic "infinite population size" approach may remain accurate over larger timescales, even in the presence of beneficial mutations, if one assumes that there is a fitness optimum, $m^{*}$. The existence of such an optimum allows for the presence of beneficial mutations (away from the optimum) but also generates saturating fitness trajectories (sublinear increase) consistent with pervasive "diminishing returns" epistasis as observed in long term evolution experiments (e.g. reviewed in Couce and Tenaillon, 2015). In such case, by definition, beneficial mutations cannot go beyond the optimal fitness. Thus, necessarily, the mutation kernel must depend on the current fitness state. The term $J \star p$ in (1) should then be replaced e.g. by

$\int_{\mathbb{R}} J_{y}(m-y) p(t, y) d y$, where $J_{y}$ is the mutation kernel, given the fitness $y$ of the parent. The mathematical investigation of integro-differential equations involving this type of mutation kernels, whose effect cannot be summarized by a convolution product, remains an open and challenging question.

\section{Proofs}

Section 4.1 is devoted to the proof of Theorem 2.2 on the global existence of solutions of (1). The proof of Proposition 2.4 on the qualitative properties of the support of $p(t, \cdot)$ in the purely deleterious case is done in Section 4.2. The explicit formula for the cumulant generating function $C(t, z)$ is proved in Section 4.3 and the proofs of Theorems 2.8 and 2.10 on the large time behaviour of the solutions of (1) and of their mean fitness and variance are carried out in Section 4.4. Lastly, Section 4.5 is devoted to the proof of some technical lemmas of the preceding sections.

\subsection{Global existence: proof of Theorem 2.2}

In order to show Theorem 2.2 on the global existence of solutions of (1), the general strategy consists in applying Cauchy-Lipschitz theorem in some suitably chosen function space. To do so, we first prove the local existence, with an existence time which is quantitatively defined in terms of the kernel $J$ and the initial probability density $p_{0}$.

Proposition 4.1 (Local existence) Let $\beta \geq 1$, let $J \in L^{1}(\mathbb{R})$ satisfy assumptions (6)-(7) and let $p_{0} \in L^{\infty}(\mathbb{R})$ satisfy (4) and

$$
0<K=\operatorname{ess~sup}_{m \in \mathbb{R}}\left(p_{0}(m) \mathrm{e}^{\beta|m|}\right)<+\infty .
$$

Let

$$
T=\min \left\{\frac{\beta}{2},\left(U \int_{\mathbb{R}} J(m) \mathrm{e}^{3 \beta|m| / 2} d m+15 K \mathrm{e}\right)^{-1}\right\}>0 .
$$


Then problem $(1)$ admits a solution $p \in \mathcal{C}^{1}\left([0, T], L^{\infty}(\mathbb{R}) \cap L^{1}(\mathbb{R})\right)$ such that $\bar{m} \in \mathcal{C}([0, T])$ and $p$ decays at least like $\mathrm{e}^{t / T-\beta|m|+t m}$ as $|m| \rightarrow+\infty$, in the sense that

$$
0 \leq p(t, m) \leq K \mathrm{e}^{t / T-\beta|m|+t m} \leq K \mathrm{e}^{1-\beta|m| / 2} \leq K \mathrm{e}^{1-T|m|} \text { for all } t \in[0, T] \text { and a.e. } m \in \mathbb{R} .
$$

Furthermore, this solution is unique.

Proof. Step 1: an auxiliary problem. Let $\beta, J, p_{0}$ and $K$ be defined as in the statement. We first show the local existence, for some well chosen $T>0$, of a solution $v$ of the following nonlinear Cauchy problem ${ }^{3}$

$$
\left\{\begin{aligned}
\partial_{t} v & =U\left(J_{t} \star v-v\right)-\bar{m}_{v}(t) v, & & t \in[0, T], m \in \mathbb{R}, \\
v(0, m) & =p_{0}(m), & & m \in \mathbb{R},
\end{aligned}\right.
$$

with

$$
J_{t}(m)=\mathrm{e}^{-t m} J(m)
$$

and

$$
\bar{m}_{v}(t)=\int_{\mathbb{R}} m \mathrm{e}^{t m} v(t, m) d m .
$$

To so so, let us first pick any real number $T$ such that $0<T \leq \beta / 2$, and let us consider the function space

$$
\begin{aligned}
& E=\left\{f \in \mathcal{C}^{1}\left([0, T], L^{\infty}(\mathbb{R})\right) \text { such that } f(0, \cdot)=p_{0}\right. \text { and } \\
& \left.\qquad 0 \leq f(t, m) \leq K \mathrm{e}^{t / T-\beta|m|} \text { for all } t \in[0, T] \text { and a.e. } m \in \mathbb{R}\right\},
\end{aligned}
$$

and denote d $^{4}$

$$
\|f\|_{E}=\left\|f(t, m) \mathrm{e}^{-t / T+\beta|m|}\right\|_{L^{\infty}([0, T] \times \mathbb{R})} .
$$

Let us now define a map $F$ as follows:

$$
\begin{aligned}
F: E & \rightarrow \mathcal{C}^{1}\left([0, T], L^{\infty}(\mathbb{R})\right), \\
v & \mapsto h,
\end{aligned}
$$

where $h=F(v)$ is the solution of the following linear Cauchy problem

$$
\left\{\begin{aligned}
\partial_{t} h & =U\left(J_{t} \star h-h\right)-\bar{m}_{v}(t) h, & & t \in[0, T], m \in \mathbb{R}, \\
h(0, m) & =p_{0}(m), & & m \in \mathbb{R} .
\end{aligned}\right.
$$

Notice that, since $v \in E$ and $0<T \leq \beta / 2$, the function $\bar{m}_{v}$ defined in (21) exists and belongs to $\mathcal{C}([0, T])$. Furthermore, Lemma 4.2 bellow affirms that $h$ is well defined as well.

Lemma 4.2 For any given $v \in E$ with $0<T \leq \beta / 2$, the Cauchy problem (22) admits a unique solution $h \in \mathcal{C}^{1}\left([0, T], L^{\infty}(\mathbb{R})\right)$.

\footnotetext{
${ }^{3}$ The equalities in (19) (as for problems (22), (29) and (38) below) are always understood for a.e. $m \in \mathbb{R}$.

${ }^{4}$ With a slight abuse of notation, we also use this notation for functions which are not necessarily in $E$.
} 
In order not to slow down the proof of Proposition 4.1, the proof of Lemma 4.2 is postponed in Section 4.5.

Step 2: $F$ maps $E$ to $E$. Consider any function $v \in E$. Since $0 \leq v(t, m) \leq K \mathrm{e}^{t / T-\beta|m|}$ for all $t \in[0, T]$ and a.e. $m \in \mathbb{R}$, it follows that, for all $t \in[0, T]$,

$$
\begin{aligned}
\left|\bar{m}_{v}(t)\right| \leq \int_{\mathbb{R}}|y| \mathrm{e}^{t y} v(t, y) d y & \leq K \int_{-\infty}^{0}|y| \mathrm{e}^{t y} \mathrm{e}^{t / T+\beta y} d y+K \int_{0}^{+\infty}|y| \mathrm{e}^{t y} \mathrm{e}^{t / T-\beta y} d y \\
& =K \mathrm{e}^{t / T}\left(\frac{1}{(\beta+t)^{2}}+\frac{1}{(\beta-t)^{2}}\right) .
\end{aligned}
$$

Consequently, we get that

$$
\left|\bar{m}_{v}(t)\right| \leq 5 K \text { e for all } 0 \leq t \leq T
$$

since $T \leq \beta / 2$ and $\beta \geq 1$

Now, set

$$
h^{+}(t, m)=K \mathrm{e}^{t / T+\beta m} \text { and } h^{-}(t, m)=K \mathrm{e}^{t / T-\beta m} \text { for }(t, m) \in[0, T] \times \mathbb{R} .
$$

We observe that, for any $(t, m) \in[0, T] \times \mathbb{R}$,

$$
\begin{aligned}
U\left(J_{t} \star h^{+}(t, m)-h^{+}(t, m)\right) & =U\left(\int_{\mathbb{R}} J(y) \mathrm{e}^{-t y} h^{+}(t, m-y) d y-h^{+}(t, m)\right) \\
& =U h^{+}(t, m)\left(\int_{\mathbb{R}} J(y) \mathrm{e}^{-(\beta+t) y} d y-1\right) .
\end{aligned}
$$

Using the assumption (6) and the inequality $T \leq \beta / 2$, we obtain that

$$
U\left(J_{t} \star h^{+}(t, m)-h^{+}(t, m)\right) \leq U h^{+}(t, m) \int_{\mathbb{R}} J(y) \mathrm{e}^{(\beta+t)|y|} d y \leq U h^{+}(t, m) \int_{\mathbb{R}} J(y) \mathrm{e}^{3 \beta|y| / 2} d y
$$

for all $(t, m) \in[0, T] \times \mathbb{R}$. Similarly,

$$
U\left(J_{t} \star h^{-}(t, m)-h^{-}(t, m)\right) \leq U h^{-}(t, m) \int_{\mathbb{R}} J(y) \mathrm{e}^{3 \beta|y| / 2} d y
$$

for all $(t, m) \in[0, T] \times \mathbb{R}$.

Consider now any real number $T$ such that

$$
0<T \leq \min \left\{\frac{\beta}{2},\left(U \int_{\mathbb{R}} J(y) \mathrm{e}^{3 \beta|y| / 2} d y+5 K \mathrm{e}\right)^{-1}\right\}
$$

Therefore, using the inequalities (23), (24) and (25), we get that

$$
\partial_{t} h^{+}=\frac{h^{+}}{T} \geq U\left(J_{t} \star h^{+}-h^{+}\right)+\left|\bar{m}_{v}\right| h^{+} \text {in }[0, T] \times \mathbb{R}
$$

and

$$
\partial_{t} h^{-}=\frac{h^{-}}{T} \geq U\left(J_{t} \star h^{-}-h^{-}\right)+\left|\bar{m}_{v}\right| h^{-} \text {in }[0, T] \times \mathbb{R} .
$$


Then, define

$$
h^{\star}(t, m)=\min \left(h^{+}(t, m), h^{-}(t, m)\right)=K \mathrm{e}^{t / T-\beta|m|} \text { for }(t, m) \in[0, T] \times \mathbb{R} .
$$

Observe that $h^{\star} \in \mathcal{C}^{1}\left([0, T], L^{\infty}(\mathbb{R})\right)$. For all $t \in[0, T]$ and $m \leq 0$, we have

$$
\partial_{t} h^{\star}(t, m)=\partial_{t} h^{+}(t, m)=\frac{h^{+}(t, m)}{T}=\frac{h^{\star}(t, m)}{T}
$$

and, as $h^{\star}(t, m)=h^{+}(t, m)$ and $h^{\star}(t, \cdot) \leq h^{+}(t, \cdot)$ in $\mathbb{R}$, we infer from (26) and (27) that

$$
\partial_{t} h^{\star}(t, m) \geq U\left(J_{t} \star h^{\star}(t, m)-h^{\star}(t, m)\right)+\left|\bar{m}_{v}(t)\right| h^{\star}(t, m) \text { for all } t \in[0, T] \text { and } m \leq 0 .
$$

Using a similar argument with $m>0$, we finally get that

$$
\partial_{t} h^{\star}=\frac{h^{\star}}{T} \geq U\left(J_{t} \star h^{\star}-h^{\star}\right)+\left|\bar{m}_{v}(t)\right| h^{\star} \geq U\left(J_{t} \star h^{\star}-h^{\star}\right)-\bar{m}_{v}(t) h^{\star} \text { in }[0, T] \times \mathbb{R} .
$$

In other words, $h^{\star} \in \mathcal{C}^{1}\left([0, T], L^{\infty}(\mathbb{R})\right)$ is a supersolution of problem $(22)$ satisfied by $h=F(v)$. From the definition (17) of $K$, we also know that $0 \leq h(0, m)=p_{0}(m) \leq h^{\star}(0, m)$ for a.e. $m \in \mathbb{R}$.

In order to conclude that $h \in E$, we will apply the following comparison principle, whose proof in postponed in Section 4.5:

Lemma 4.3 (Comparison principle) ${ }^{5}$ Let $\tau \in(0,+\infty), \bar{m} \in \mathcal{C}([0, \tau])$ and $h_{1}, h_{2} \in$ $\mathcal{C}^{1}\left([0, \tau], L^{\infty}(\mathbb{R})\right)$ be such that, for every $t \in[0, \tau]$,

$$
\partial_{t} h_{1}-U\left(J_{t} \star h_{1}-h_{1}\right)+\bar{m}(t) h_{1} \leq \partial_{t} h_{2}-U\left(J_{t} \star h_{2}-h_{2}\right)+\bar{m}(t) h_{2}
$$

for a.e. $m \in \mathbb{R}$, with $J_{t}$ defined in (20). Assume that $h_{1}(0, m) \leq h_{2}(0, m)$ for a.e. $m \in \mathbb{R}$ at initial time. Then, for every $t \in[0, \tau], h_{1}(t, m) \leq h_{2}(t, m)$ for a.e. $m \in \mathbb{R}$.

Thus, we obtain that, for every $t \in[0, T], 0 \leq h(t, m) \leq h^{\star}(t, m)$ for a.e. $m \in \mathbb{R}$. Together with Lemma 4.2, it follows that $h=F(v) \in E$.

Step 3: $F$ is a contraction mapping. Let $v_{1}, v_{2} \in E$ and define $H=F\left(v_{1}\right)-F\left(v_{2}\right)$. We note that the function $H$ belongs to $\mathcal{C}^{1}\left([0, T], L^{\infty}(\mathbb{R})\right)$ and satisfies

$$
\left\{\begin{aligned}
\partial_{t} H & =U\left(J_{t} \star H-H\right)-\left(\bar{m}_{v_{1}}-\bar{m}_{v_{2}}\right) F\left(v_{1}\right)-\bar{m}_{v_{2}} H, & & t \in[0, T], m \in \mathbb{R}, \\
H(0, m) & =0, & & m \in \mathbb{R} .
\end{aligned}\right.
$$

Define

$$
H^{+}(t, m)=\frac{\left\|v_{1}-v_{2}\right\|_{E}}{2} \mathrm{e}^{t / T+\beta m} \text { and } H^{-}(t, m)=\frac{\left\|v_{1}-v_{2}\right\|_{E}}{2} \mathrm{e}^{t / T-\beta m}
$$

for $(t, m) \in[0, T] \times \mathbb{R}$. Similar computations as for $h^{ \pm}$and $v$ above imply that:

$$
U\left(J_{t} \star H^{ \pm}-H^{ \pm}\right) \leq U H^{ \pm} \int_{\mathbb{R}} J(y) \mathrm{e}^{3 \beta|y| / 2} d y \text { in }[0, T] \times \mathbb{R}
$$

\footnotetext{
${ }^{5}$ This lemma is adapted from Lemma 15 in Yagisita (2009), where a comparison principle was obtained for equations of the form $\partial_{t} v=J \star v-v+g(v)$. Here, $g(v)$ is replaced by a time-dependent term $-\bar{m}(t) v$.
} 
and

$$
\left|\bar{m}_{v_{1}}(t)-\bar{m}_{v_{2}}(t)\right| \leq 5 \mathrm{e}\left\|v_{1}-v_{2}\right\|_{E} \text { for all } t \in[0, T] .
$$

Since $F\left(v_{1}\right) \in E$, it follows that

$$
\left|\left(\bar{m}_{v_{1}}(t)-\bar{m}_{v_{2}}(t)\right) F\left(v_{1}\right)\right| \leq 5 K \mathrm{e}\left\|v_{1}-v_{2}\right\|_{E} \mathrm{e}^{t / T-\beta|m|} \leq 10 K \text { e } H^{ \pm} \text {in }[0, T] \times \mathbb{R} .
$$

Finally, let us consider the real number $T$ defined by (18). Using (23), (30) and (31), we get that

$$
\partial_{t} H^{ \pm}=\frac{H^{ \pm}}{T} \geq U\left(J_{t} \star H^{ \pm}-H^{ \pm}\right)+\left|\left(\bar{m}_{v_{1}}(t)-\bar{m}_{v_{2}}(t)\right) F\left(v_{1}\right)\right|+\left|\bar{m}_{v_{2}}(t)\right| H^{ \pm} \text {in }[0, T] \times \mathbb{R} .
$$

As above, this implies that $H^{\star}=\min \left(H^{+}, H^{-}\right)$is a supersolution of equation (29) satisfied by $H$, while $H^{\star} \in \mathcal{C}^{1}\left([0, T], L^{\infty}(\mathbb{R})\right)$. Using again the comparison principle in Lemma 4.3, we get that

$$
H(t, m) \leq H^{\star}(t, m) \text { for all } t \in[0, T] \text { and a.e. } m \in \mathbb{R}
$$

and, similarly, $-H(t, m) \leq H^{\star}(t, m)$ for all $t \in[0, T]$ and a.e. $m \in \mathbb{R}$. This immediately implies that

$$
\left\|F\left(v_{1}\right)-F\left(v_{2}\right)\right\|_{E} \leq \frac{1}{2}\left\|v_{1}-v_{2}\right\|_{E}
$$

Thus $F$ is a contraction mapping.

Step 4: conclusion. With $T>0$ defined by (18), Banach fixed point theorem implies that $F$ admits a unique fixed point $v \in E$. This function $v$ belongs to $\mathcal{C}^{1}\left([0, T], L^{\infty}(\mathbb{R})\right)$, is such that

$$
0 \leq v(t, m) \leq K \mathrm{e}^{t / T-\beta|m|} \text { for all } t \in[0, T] \text { and a.e. } m \in \mathbb{R},
$$

and it is the unique such solution of (19).

Finally, letting

$$
p(t, m)=\mathrm{e}^{t m} v(t, m)
$$

for $(t, m) \in[0, T] \times \mathbb{R}$, it is straightforward to check that $p \in \mathcal{C}^{1}\left([0, T], L^{\infty}(\mathbb{R})\right)$ is a solution of (1) for $t \in[0, T]$. Additionally, as $v \in E$ and $T \leq \beta / 2$, it follows that $p(t, \cdot) \in L^{1}(\mathbb{R})$ for all $t \in[0, T]$, that $p \in \mathcal{C}^{1}\left([0, T], L^{\infty}(\mathbb{R}) \cap L^{1}(\mathbb{R})\right)$, that $t \mapsto \bar{m}(t)=\int_{\mathbb{R}} m p(t, m) d m \in \mathcal{C}([0, T])$ and that

$$
0 \leq p(t, m) \leq K \mathrm{e}^{t / T-\beta|m|+t m} \text { for all } t \in[0, T] \text { and a.e. } m \in \mathbb{R} .
$$

The proof of Proposition 4.1 is thereby complete.

Proof of Theorem 2.2. We are now in position to prove the global existence result of Theorem 2.2. Let $T_{\max }>0$ be the largest time such that equation (1) admits a solution $p \in \mathcal{C}^{1}\left(\left[0, T_{\max }\right), L^{\infty}(\mathbb{R}) \cap L^{1}(\mathbb{R})\right)$ with $\bar{m} \in \mathcal{C}\left(\left[0, T_{\max }\right)\right)$ and, for all $T \in\left(0, T_{\max }\right)$, there is $C_{T}>0$ such that

$$
0 \leq p(t, m) \leq C_{T} \mathrm{e}^{-T|m|} \text { for all } t \in[0, T] \text { and a.e. } m \in \mathbb{R} .
$$

It follows from the local existence and uniqueness result of Proposition 4.1 applied with, say, $\beta=1$ that

$$
T_{\text {max }} \in(0,+\infty] \text {. }
$$

Notice that $T_{\max }$ does not depend on the choice $\beta=1$ in Proposition 4.1, it is characterized by (32) only. Our goal is to show that $T_{\max }=+\infty$.

We begin with some fundamental estimates (whose proof is postponed in Section 4.5): 
Lemma 4.4 (Mass preservation and estimates on the mean fitness) We have:

$$
\int_{\mathbb{R}} p(t, m) d m=1 \text { for all } t \in\left[0, T_{\max }\right)
$$

and

$$
\bar{m}(t) \geq \bar{m}(0)+t U \mu_{J} \text { for all } t \in\left[0, T_{\max }\right),
$$

with $\mu_{J}=\int_{\mathbb{R}} m J(m) d m$.

Assume now by contradiction that $T_{\max }<+\infty$. Define

$$
v(t, m)=p(t, m) \mathrm{e}^{-t m}
$$

for $(t, m) \in\left[0, T_{\max }\right) \times \mathbb{R}$. The function $v$ satisfies (19) for all $T \in\left[0, T_{\max }\right)$ and, from (32) and the regularity properties of $p$, the function $v$ belongs to $\mathcal{C}^{1}\left(\left[0, T_{\max }\right), L^{\infty}(\mathbb{R})\right)$. Set now

$$
\beta_{0}=2 T_{\max }+1
$$

and let

$$
K_{0}=\operatorname{ess}_{m \in \mathbb{R}}\left(p_{0}(m) \mathrm{e}^{\beta_{0}|m|}\right)
$$

be defined as in (17) in Proposition 4.1 with this choice $\beta=\beta_{0}$. Denote

$$
v^{ \pm}(t, m)=K_{0} \mathrm{e}^{\lambda t \pm \beta_{0} m}
$$

for $(t, m) \in\left[0, T_{\max }\right) \times \mathbb{R}$, where $\lambda \in \mathbb{R}$ is to be chosen later. Using (34) and the property $\bar{m}(t)=$ $\bar{m}_{v}(t)$ for all $t \in\left[0, T_{\max }\right)$, it is easily seen (as in Step 2 of the proof of Proposition 4.1) that, for every $T \in\left(0, T_{\max }\right)$, the function $v^{\star}=\min \left(v^{+}, v^{-}\right)$is a supersolution of the equation (19) (for which $\bar{m}_{v}(t)=\bar{m}(t)$ is considered as a fixed coefficient) satisfied by $v$ on $[0, T]$, provided that

$$
\lambda \geq U \int_{\mathbb{R}} J(m) \mathrm{e}^{3 \beta_{0}|m| / 2} d m-\bar{m}(0)+T U\left|\mu_{J}\right| .
$$

Let then

$$
\lambda^{\star}=U \int_{\mathbb{R}} J(m) \mathrm{e}^{3 \beta_{0}|m| / 2} d m+|\bar{m}(0)|+T_{\max } U\left|\mu_{J}\right| \geq 0 .
$$

Using the comparison principle of Lemma 4.3 applied with every $\tau \in\left(0, T_{\max }\right)$, we obtain that

$$
0 \leq v(t, m) \leq K_{0} \mathrm{e}^{\lambda^{\star} t-\beta_{0}|m|} \text { for all } t \in\left[0, T_{\max }\right) \text { and a.e. } m \in \mathbb{R} .
$$

Now, for any $\theta \in(0,1)$, set $p_{\theta}=p\left(\theta T_{\max }, \cdot\right)$. We have

$$
p_{\theta}(m)=v\left(\theta T_{\max }, m\right) \mathrm{e}^{\theta T_{\max } m} \leq K_{0} \mathrm{e}^{\lambda^{\star} T_{\max }+\left(T_{\max }-\beta_{0}\right)|m|}=K_{0} \mathrm{e}^{\lambda^{\star} T_{\max }-\left(T_{\max }+1\right)|m|}
$$

for a.e. $m \in \mathbb{R}$. For any $\theta \in(0,1)$, the function $p_{\theta}$ satisfies (4), owing to (32) and (33). Furthermore,

$$
0<K_{\theta}:=\operatorname{ess}_{m \in \mathbb{R}}\left(p_{\theta}(m) \mathrm{e}^{\left(T_{\max }+1\right)|m|}\right) \leq K_{0} \mathrm{e}^{\lambda^{\star} T_{\max }}<+\infty .
$$


As a consequence, we can apply Proposition 4.1 with

$$
\beta=T_{\max }+1
$$

and the initial condition $p_{\theta}$. Thus, for any $\theta \in(0,1)$, there exist a time $T_{\theta}>0$, defined as in (18) with $K_{\theta}$ instead of $K$, and a unique solution $\tilde{p} \in \mathcal{C}^{1}\left(\left[0, T_{\theta}\right], L^{\infty}(\mathbb{R}) \cap L^{1}(\mathbb{R})\right)$ of (1) with initial condition $p_{\theta}$, such that

$$
0 \leq \tilde{p}(t, m) \leq K_{\theta} \mathrm{e}^{t / T_{\theta}-\left(T_{\max }+1\right)|m|+t m}
$$

for all $t \in\left[0, T_{\theta}\right]$ and a.e. $m \in \mathbb{R}$. Therefore, for any $\theta \in(0,1)$, problem (1) with initial condition $p_{0}$ has a solution $p \in \mathcal{C}^{1}\left(\left[0, \theta T_{\max }+T_{\theta}\right], L^{\infty}(\mathbb{R}) \cap L^{1}(\mathbb{R})\right)$ such that, for all $t \in$ $\left[\theta T_{\max }, \theta T_{\max }+\min \left(T_{\theta}, 1 / 2\right)\right]$ and a.e. $m \in \mathbb{R}$,

$$
0 \leq p(t, m) \leq K_{\theta} \mathrm{e}^{\left(t-\theta T_{\max }\right) / T_{\theta}-\left(T_{\max }+1\right)|m|+\left(t-\theta T_{\max }\right) m} \leq K_{0} \mathrm{e}^{\lambda^{\star} T_{\max }+1-\left(T_{\max }+1 / 2\right)|m|} .
$$

On the other hand, from (36) and the definition (18) of $T_{\theta}>0$ with $K_{\theta}$ instead of $K$, it follows that $\liminf _{\theta \rightarrow 1} T_{\theta}>0$. Therefore, there exist $\theta^{\prime} \in(0,1)$ and $T^{\prime} \in\left(T_{\max }, T_{\max }+1 / 2\right)$ for which problem $(1)$ with initial condition $p_{0}$ has a solution $p \in \mathcal{C}^{1}\left(\left[0, T^{\prime}\right], L^{\infty}(\mathbb{R}) \cap L^{1}(\mathbb{R})\right)$ such that, for all $t \in\left[\theta^{\prime} T_{\max }, T^{\prime}\right]$ and a.e. $m \in \mathbb{R}$,

$$
0 \leq p(t, m) \leq K_{0} \mathrm{e}^{\lambda^{\star} T_{\max }+1-\left(T_{\max }+1 / 2\right)|m|} \leq K_{0} \mathrm{e}^{\lambda^{\star} T_{\max }+1-T^{\prime}|m|} .
$$

Furthermore, (35) (remember that $\beta_{0}=2 T_{\max }+1$ ) implies that, for all $t \in\left[0, \theta^{\prime} T_{\max }\right]$ and a.e. $m \in \mathbb{R}$,

$$
0 \leq p(t, m)=v(t, m) e^{t m} \leq K_{0} \mathrm{e}^{\lambda^{\star} \theta^{\prime} T_{\max }-\left(2 T_{\max }+1\right)|m|+T_{\max }|m|} \leq K_{0} \mathrm{e}^{\lambda^{\star} \theta^{\prime} T_{\max }-T^{\prime}|m|} .
$$

Together with (37) in $\left[\theta^{\prime} T_{\max }, T^{\prime}\right] \times \mathbb{R}$, it follows that the solution $p$ satisfies (32) for all $T \in\left(0, T^{\prime}\right]$. Finally, one infers that $\bar{m} \in \mathcal{C}\left(\left[0, T^{\prime}\right]\right)$. The fact that $T^{\prime}$ is larger than $T_{\max }$ contradicts the definition of $T_{\max }$.

As a conclusion, $T_{\max }=+\infty$ and, from (32) holding for any $T>0$, property (9) holds with $\Gamma_{\alpha, T}=C_{\max (\alpha, T)}$. From the equation (1) itself, it also follows that $\left|\partial_{t} p(t, m)\right|$ decays faster than any exponential function as $|m| \rightarrow+\infty$ in the sense that (9) holds for $\left|\partial_{t} p(t, m)\right|$ as well. The proof of Theorem 2.2 is thereby complete.

\subsection{Purely deleterious kernels: proof of Proposition 2.4}

Proof of Proposition 2.4. We assume that $p_{0} \in L^{\infty}(\mathbb{R})$ satisfies (4)-(5) and that $J \in L^{1}(\mathbb{R})$ satisfies (6)-(7) and is purely deleterious in the sense of (10). Let $m_{0} \in(-\infty,+\infty]$ be defined by (11). In order to show that $\operatorname{supp}(p(t, \cdot)) \subset\left(-\infty, m_{0}\right]$ for all $t \geq 0$, the main tool will be the comparison principle stated in Lemma 4.3. First of all, if $m_{0}=\sup \left\{\operatorname{supp}\left(p_{0}\right)\right\}=+\infty$, then the desired result is obviously true.

Assume now that $m_{0}<+\infty$. Without loss of generality, even if it means replacing $p(t, m)$ by $p\left(t, m+m_{0}\right)$, we can assume that $m_{0}=0$. In other words, it then follows from (4)-(5) that, for every $\alpha \geq 0$, there is $K_{\alpha}>0$ such that

$$
0 \leq p_{0}(m) \leq K_{\alpha} \mathrm{e}^{\alpha m} \mathbb{1}_{(-\infty, 0]}(m) \text { for a.e. } m \in \mathbb{R}
$$


where $\mathbb{1}_{(-\infty, 0]}$ denotes the characteristic function of the interval $(-\infty, 0]$. From Theorem 2.2, let $p \in \mathcal{C}^{1}\left([0,+\infty), L^{\infty}(\mathbb{R})\right)$ be the solution of problem (1) such that $p$ satisfies $(9)$ and $\bar{m} \in$ $\mathcal{C}([0,+\infty))$. It is straightforward to check that the function $w$ defined in $[0,+\infty) \times \mathbb{R}$ by

$$
w(t, m)=\mathrm{e}^{-t m+U t+\int_{0}^{t} \bar{m}(s) d s} p(t, m)
$$

belongs to $\mathcal{C}^{1}\left([0,+\infty), L^{\infty}(\mathbb{R})\right)$ and obeys

$$
\left\{\begin{aligned}
\partial_{t} w & =U J_{t} \star w, \quad t \geq 0, m \in \mathbb{R} \\
w(0, m) & =p_{0}(m), \quad m \in \mathbb{R}
\end{aligned}\right.
$$

where we recall that $J_{t}$ is defined in $(20)$.

Fix $T>0$. Let us now look for a nonnegative supersolution of equation (38) for $t \in[0, T]$, namely a nonnegative function $w^{+}$satisfying $w^{+}(0, \cdot) \geq p_{0}$ and

$$
\partial_{t} w^{+}(t, m) \geq U J_{t} \star w^{+}(t, m) \text { for all } t \in[0, T] \text { and } m \in \mathbb{R} .
$$

We also seek $w$ so that $\operatorname{supp}(w(t, \cdot)) \subset(-\infty, 0]$ for all $t \in[0, T]$. In that respect, we look for a function $w^{+}$of the type

$$
w^{+}(t, m)=K \mathrm{e}^{\lambda t} \mathbb{1}_{(-\infty, 0]}(m)
$$

for some positive reals $K$ and $\lambda$. Using the assumption (10), it is easily seen that $w^{+}$of the above type satisfies condition (39) if and only if

$$
\lambda \geq U \int_{-\infty}^{0} J(y) \mathrm{e}^{-T y} d y
$$

Let then

$$
\lambda^{*}=U \int_{-\infty}^{0} J(y) \mathrm{e}^{-T y} d y>0, \quad K^{*}=\underset{(-\infty, 0]}{\operatorname{ess} \sup } p_{0} \text { and } w^{+}(t, m)=K^{*} \mathrm{e}^{\lambda^{*} t} \mathbb{1}_{(-\infty, 0]}(m)
$$

for all $t \in[0, T]$ and $m \in \mathbb{R}$. Then $w^{+} \in \mathcal{C}^{1}\left([0, T], L^{\infty}(\mathbb{R})\right)$ is a supersolution of equation (38), while $w^{+}(0, \cdot) \geq p_{0}=w(0, \cdot) \geq 0$. Thus, using the comparison principle of Lemma 4.3, we obtain that $0 \leq w(t, m) \leq w^{+}(t, m)$ for all $t \in[0, T]$ and a.e. $m \in \mathbb{R}$. In particular, there holds

$$
w(t, m)=0 \text { for all } t \in[0, T] \text { and a.e. } m \in(0,+\infty) .
$$

Consequently, we get that $p(t, m)=0$ for all $t \in[0, T]$ and a.e. $m \in(0,+\infty)$. Since $T>0$ can be chosen as large as we want, the desired conclusion of Proposition 2.4 follows.

\subsection{Cumulant generating function: proof of Theorem 2.5}

Proof of Theorem 2.5. We here consider the problem:

$$
\left\{\begin{aligned}
\partial_{t} C(t, z) & =\partial_{z} C(t, z)-\partial_{z} C(t, 0)+\beta(z), & & t \geq 0, z \geq 0 \\
C(t, 0) & =0, & & t \geq 0 \\
C(0, z) & =C_{0}(z), & & z \geq 0
\end{aligned}\right.
$$


where $\beta \in \mathcal{C}([0,+\infty))$ is such that $\beta(0)=0$ and $C_{0} \in \mathcal{C}^{1}([0,+\infty))$ satisfies $C_{0}(0)=0$. In order to get formula (15) for $C$, we use the method of characteristics. Let $C \in \mathcal{C}^{1}([0,+\infty) \times[0,+\infty))$ be a solution of (40). Fix $t \geq 0, z \geq 0$, and denote

$$
v(s)=C(t+s, z-s) \text { for } s \in[-t, z] .
$$

The function $v$ belongs to $\mathcal{C}^{1}([-t, z])$ and, using (40), we have $v^{\prime}(s)=-\partial_{z} C(t+s, 0)+\beta(z-s)$ for all $s \in[-t, z]$. Thus,

$$
\begin{aligned}
C(t, z)-C(0, z+t)=v(0)-v(-t) & =\int_{-t}^{0}\left(-\partial_{z} C(t+s, 0)+\beta(z-s)\right) d s \\
& =-\int_{0}^{t} \partial_{z} C(s, 0) d s+\int_{0}^{t} \beta(z+s) d s .
\end{aligned}
$$

Hence,

$$
C(t, z)=C_{0}(z+t)+\int_{0}^{t} \beta(z+s) d s-\int_{0}^{t} \partial_{z} C(s, 0) d s \text { for all } t \geq 0 \text { and } z \geq 0 .
$$

The resulting expression still depends on the non-local term $\partial_{z} C(\cdot, 0)$. Deriving the last equation with respect to $z$, we get that

$$
\partial_{z} C(t, z)=C_{0}^{\prime}(z+t)+\beta(z+t)-\beta(z) \text { for all } t \geq 0 \text { and } z \geq 0
$$

and $\partial_{z} C(t, 0)=C_{0}^{\prime}(t)+\beta(t)$ for all $t \geq 0$. Therefore,

$$
C(t, z)=C_{0}(z+t)-C_{0}(t)+\int_{0}^{t}(\beta(z+s)-\beta(s)) d s \text { for all } t \geq 0 \text { and } z \geq 0,
$$

that is, (15) is proved.

Lastly, for the sake of completeness, let us check that the function $C$ defined by (41) obeys (40). As $\beta \in \mathcal{C}([0,+\infty))$ with $\beta(0)=0$ and $C_{0} \in \mathcal{C}^{1}([0,+\infty))$ with $C_{0}(0)=0$, we know that $C \in \mathcal{C}^{1}([0,+\infty) \times[0,+\infty))$ and

$$
\left\{\begin{array}{l}
\partial_{z} C(t, z)=C_{0}^{\prime}(z+t)+\beta(z+t)-\beta(z) \\
\partial_{t} C(t, z)=C_{0}^{\prime}(z+t)-C_{0}^{\prime}(t)+\beta(z+t)-\beta(t)
\end{array} \quad \text { for all } t \geq 0 \text { and } z \geq 0 .\right.
$$

Therefore, $\partial_{z} C(t, 0)=C_{0}^{\prime}(t)+\beta(t)$, whence $\partial_{t} C(t, z)=\partial_{z} C(t, z)-\partial_{z} C(t, 0)+\beta(z)$. Moreover $C(t, 0)=0$ and $C(0, z)=C_{0}(z)-C_{0}(0)=C_{0}(z)$ for all $t \geq 0$ and $z \geq 0$. In other words, $C$ is a solution of $(40)$.

Finally, this shows that (40) admits a unique solution $C \in \mathcal{C}^{1}([0,+\infty) \times[0,+\infty))$, and that it is given by (41). The proof of Theorem 2.5 is thereby complete.

\subsection{Large time behaviour: proof of Theorems 2.8 and 2.10}

We first deal with the proof of Theorem 2.8 on the large time behaviour of the mean fitness and the variance in fitness. We then do the proof of Theorem 2.10 on the limiting fitness distribution in the purely deleterious case. Both proofs are based on asymptotic properties of 
the initial cumulant generating function $C_{0}=C(0, \cdot)$ and its derivatives.

Proof of Theorem 2.8. In Corollary 2.6, we stated that the mean fitness and the variance in fitness were given by

$$
\left\{\begin{array}{l}
\bar{m}(t)=C_{0}^{\prime}(t)+\beta(t) \\
V(t)=C_{0}^{\prime \prime}(t)+\beta^{\prime}(t)-\beta^{\prime}(0)
\end{array}\right.
$$

for all $t \geq 0$, where the functions $\beta$ and $C_{0}$ are given by (14). We are interested here in the limits of $\bar{m}(t)$ and $V(t)$ as $t \rightarrow+\infty$.

Firstly, the limits of the derivatives of the CGF $C_{0}$ are given by the following lemma below, whose proof is postponed in Section 4.5.

Lemma 4.5 Let $m_{0} \in(-\infty,+\infty]$ be defined by (11). The function $C_{0}$ is convex and $C_{0}^{\prime}(t) \rightarrow$ $m_{0}$ as $t \rightarrow+\infty$. Furthermore, if $m_{0}<+\infty$, then

$$
\lim _{t \rightarrow+\infty} C_{0}^{\prime \prime}(t)=0
$$

Secondly, we observe that, from the properties of $J$, there holds

$$
\beta(t)=U \int_{\mathbb{R}} J(m) \mathrm{e}^{t m} d m-U \text { and } \beta^{\prime}(t)=U \int_{\mathbb{R}} m J(m) \mathrm{e}^{t m} d m \text { for all } t \geq 0
$$

and, in particular,

$$
\beta^{\prime}(0)=U \int_{\mathbb{R}} m J(m) d m=U \mu_{J}
$$

Let us now prove assertion (i) of Theorem 2.8. We assume here that $\operatorname{supp}(J) \subset(-\infty, 0]$ and $m_{0}<+\infty$. Then $\beta(t)=U \int_{-\infty}^{0} J(m) \mathrm{e}^{t m} d m-U$ for all $t \geq 0$ and, since $J \in L^{1}(\mathbb{R})$, Lebesgue's dominated convergence theorem yields $\lim _{t \rightarrow+\infty} \beta(t)=-U$. Similarly, we obtain that $\lim _{t \rightarrow+\infty} \beta^{\prime}(t)=0$. Therefore, using Lemma 4.5 and (44), we finally obtain that

$$
\left\{\begin{aligned}
\lim _{t \rightarrow+\infty} \bar{m}(t) & =\lim _{t \rightarrow+\infty} C_{0}^{\prime}(t)+\lim _{t \rightarrow+\infty} \beta(t)=m_{0}-U<m_{0}, \\
\lim _{t \rightarrow+\infty} V(t) & =\lim _{t \rightarrow+\infty} C_{0}^{\prime \prime}(t)+\lim _{t \rightarrow+\infty} \beta^{\prime}(t)-\beta^{\prime}(0)=-\beta^{\prime}(0)=-U \mu_{J}>0,
\end{aligned}\right.
$$

with, here, $\mu_{J}=\int_{-\infty}^{0} m J(m) d m<0$.

Let us now deal with property (ii) of Theorem 2.8. Thus, we assume in this paragraph that $\operatorname{supp}(J) \subset(-\infty, 0]$ and $m_{0}=+\infty$. It follows again from the finiteness of the limit $\lim _{t \rightarrow+\infty} \beta(t)=-U$ and from Lemma 4.5 that $\lim _{t \rightarrow+\infty} \bar{m}(t)=+\infty$. Lastly, the formula $V(+\infty)=C_{0}^{\prime \prime}(+\infty)-U \mu_{J}$, when $p_{0}$ is such that the limit $C_{0}^{\prime \prime}(+\infty)$ is well-defined, is a direct consequence of the computations of part (i) and of (42).

Let us now turn to the proof of assertion (iii) of Theorem 2.8. We assume here that $\operatorname{supp}(J) \nsubseteq(-\infty, 0]$. That is, there are a non-negligible measurable set $A \subset(0,+\infty)$ and $\alpha>0$ such that

$$
J(m)>\alpha \text { for all } m \in A .
$$

Then, as $J(m) \mathrm{e}^{t m} \geq 0$ for all $t \geq 0$ and $m \in \mathbb{R}$, it follows that

$$
\beta(t)=U \int_{\mathbb{R}} J(m) \mathrm{e}^{t m} d m-U \geq U \int_{A} J(m) \mathrm{e}^{t m} d m-U \geq \alpha U \int_{A} \mathrm{e}^{t m} d m-U \underset{t \rightarrow+\infty}{\rightarrow}+\infty .
$$


Using Lemma 4.5, we conclude that

$$
\lim _{t \rightarrow+\infty} \bar{m}(t)=\lim _{t \rightarrow+\infty} C_{0}^{\prime}(t)+\lim _{t \rightarrow+\infty} \beta(t)=+\infty
$$

Similarly,

$$
\begin{aligned}
\beta^{\prime}(t)=U \int_{\mathbb{R}} m J(m) \mathrm{e}^{t m} d m & =U \int_{-\infty}^{0} m J(m) \mathrm{e}^{t m} d m+U \int_{0}^{+\infty} m J(m) \mathrm{e}^{t m} d m \\
& \geq-U \int_{-\infty}^{0}|m| J(m) d m+\alpha U \int_{A} m \mathrm{e}^{t m} d m \underset{t \rightarrow+\infty}{\rightarrow}+\infty .
\end{aligned}
$$

Hence, since $C_{0}$ is convex, it follows from Corollary 2.6 that $\lim _{t \rightarrow+\infty} V(t)=+\infty$. The proof of Theorem 2.8 is thereby complete.

Proof of Theorem 2.10. Let us first assume that $m_{0}$ given in (11) satisfies $m_{0}=0$. Using Theorem 2.5, we have

$$
C(t, z)=C_{0}(t+z)-C_{0}(t)+\int_{0}^{t}(\beta(z+s)-\beta(s)) d s \text { for all } t \geq 0 \text { and } z \geq 0 .
$$

As $C_{0} \in \mathcal{C}^{1}([0,+\infty))$, it follows that, for any $t \geq 0$ and $z \geq 0$, there is $x_{t, z} \in[t, t+z]$ such that $C_{0}(t+z)-C_{0}(t)=z C_{0}^{\prime}\left(x_{t, z}\right)$. For any given $z \geq 0$, as $\lim _{t \rightarrow+\infty} x_{t, z}=+\infty$, Lemma 4.5 yields

$$
\lim _{t \rightarrow+\infty}\left(C_{0}(t+z)-C_{0}(t)\right)=\lim _{t \rightarrow+\infty} z C_{0}^{\prime}\left(x_{t, z}\right)=0 .
$$

Moreover, for any $z \geq 0$ and $t \geq z$, one has

$$
\begin{aligned}
\int_{0}^{t}(\beta(z+s)-\beta(s)) d s & =\int_{z}^{z+t} \beta(s) d s-\int_{0}^{t} \beta(s) d s=\int_{t}^{z+t} \beta(s) d s-\int_{0}^{z} \beta(s) d s \\
& =U \int_{t}^{z+t} \int_{-\infty}^{0} J(y) \mathrm{e}^{s y} d y d s-U \int_{0}^{z} \int_{-\infty}^{0} J(y) \mathrm{e}^{s y} d y d s \\
& =U \int_{0}^{z} \int_{-\infty}^{0} J(y) \mathrm{e}^{(s+t) y} d y d s-U \int_{0}^{z} \int_{-\infty}^{0} J(y) \mathrm{e}^{s y} d y d s .
\end{aligned}
$$

Lebesgue's dominated convergence theorem implies that the first integral converges to 0 as $t \rightarrow+\infty$. Therefore, for any $z \geq 0, C(t, z)$ admits a limit $C_{\infty}(z) \in \mathbb{R}$ as $t \rightarrow+\infty$, with

$$
C_{\infty}(z)=-U \int_{0}^{z} \int_{-\infty}^{0} J(y) \mathrm{e}^{s y} d y d s=U \int_{-\infty}^{0} \frac{J(y)}{y}\left(1-\mathrm{e}^{z y}\right) d y .
$$

Obviously, $C_{\infty}$ is continuous at 0 . Thus, using Theorem 2.9 , the family $(p(t, .))_{t \geq 0}$ converges weakly as $t \rightarrow+\infty$ to a nonnegative measure $p_{\infty}$ such that

$$
C_{\infty}(z)=\ln \left(\int_{\mathbb{R}} \mathrm{e}^{z m} d p_{\infty}(m)\right) \text { for all } z \geq 0 .
$$

Using Proposition 2.4, we know that the support of $p(t, \cdot)$ is included in $(-\infty, 0]$ for all $t \geq 0$, and that property holds for $p_{\infty}$ as well. Therefore we can write

$$
p_{\infty}=\rho \delta_{0}+(1-\rho) p^{\star}
$$


where $\delta_{0}$ is the Dirac measure at 0 and $p^{\star}$ is a nonnegative measure supported in $(-\infty, 0]$ and having no mass at 0 , that is, $p^{\star}([-\varepsilon, 0]) \rightarrow 0$ as $\varepsilon \rightarrow 0$. Furthermore, since $C_{\infty}(0)=0$ by (45), it follows from (46) that $p_{\infty}$ has unit mass, hence $p^{\star}$ has unit mass too.

In order to compute the weight $\rho$ of the mass at 0 , we pass to the limit as $z \rightarrow+\infty$ in formulas (45) and (46). As $J$ is nonnegative, equation (45) and the monotone convergence theorem lead to

$$
\lim _{z \rightarrow+\infty} C_{\infty}(z)=U \int_{-\infty}^{0} \frac{J(y)}{y} d y \in[-\infty, 0) .
$$

Then, using (46) and (47), we get that

$$
C_{\infty}(z)=\ln \left(\int_{\mathbb{R}} \mathrm{e}^{z m} d p_{\infty}(m)\right)=\ln \left(\rho+(1-\rho) \int_{-\infty}^{0} \mathrm{e}^{z m} d p^{\star}(m)\right) .
$$

As $p^{\star}$ has no mass at 0 , it follows that $\lim _{z \rightarrow+\infty} C_{\infty}(z)=\ln (\rho)$. Finally, we obtain that

$$
\rho=\exp \left(U \int_{-\infty}^{0} \frac{J(y)}{y} d y\right) \in[0,1),
$$

with the convention $\exp (-\infty)=0$. Remember that the harmonic mean $-s_{H}$ of $J$ is defined by $-s_{H}=\left(\int_{-\infty}^{0} \frac{J(y)}{y} d y\right)^{-1} \in(-\infty, 0]$. Therefore, on the one hand, if $s_{H}=0$, i.e. $\int_{-\infty}^{0} \frac{J(y)}{y} d y=$ $-\infty$, then $\rho=0$. On the other hand, if $s_{H} \neq 0$, then $\rho=\mathrm{e}^{-U / s_{H}} \in(0,1)$.

Finally, consider the general case $m_{0} \in \mathbb{R}$ and let $q(t, m)=p\left(t, m+m_{0}\right)$. It follows from Proposition 2.4 that, for every $t \geq 0$, the support of $q(t, \cdot)$ is included in $(-\infty, 0]$. Let $C^{q}$ be the CGF associated with $q$. We have

$$
C(t, z)=C^{q}(t, z)+m_{0} z
$$

for all $t \geq 0$ and $z \geq 0$, and the nonnegative limiting measure $p_{\infty}$ of $p(t, \cdot)$ as $t \rightarrow+\infty$ can then be written as $p_{\infty}=\rho \delta_{m_{0}}+(1-\rho) p^{\star}$, where $\delta_{m_{0}}$ is the Dirac measure at $m_{0}$ and $p^{\star}$ is a nonnegative measure supported in $\left(-\infty, m_{0}\right]$ and having no mass at $m_{0}$. Formula (16) follows from (48) and from formula (45) applied to $q$. Similar arguments also imply that $\rho=\mathrm{e}^{-U / s_{H}} \in[0,1)$ and the proof of Theorem 2.10 is thereby complete.

\subsection{Proofs of the technical lemmas}

This section is devoted to the proof of the technical lemmas used in the proof of the main theorems in the previous sections. Let us begin with the

Proof of Lemma 4.2. Let $v \in E$. We know by definition of the set $E$ and Lebesgue's dominated convergence theorem that the function

$$
t \mapsto \bar{m}_{v}(t)=\int_{\mathbb{R}} m v(t, m) d m
$$

is of class $\mathcal{C}^{1}([0, T])$. Problem $(22)$ can then be written as an ordinary differential equation

$$
\left\{\begin{array}{l}
h^{\prime}(t)=G(t, h), \quad t \in[0, T], \\
h(0)=p_{0},
\end{array}\right.
$$


with

$$
\begin{aligned}
G:[0, T] \times L^{\infty}(\mathbb{R}) & \rightarrow L^{\infty}(\mathbb{R}) \\
(t, X) & \mapsto U\left(J_{t} \star X\right)-\left(U+\bar{m}_{v}(t)\right) X .
\end{aligned}
$$

The function space $L^{\infty}(\mathbb{R})$ is a Banach space for the uniform norm \|\|$_{\infty}$. As easily follows from Lebesgue's dominated convergence theorem and elementary arguments, the function $G$ is continuous on $[0, T] \times L^{\infty}(\mathbb{R})$. It is also uniformly Lipschitz-continuous with respect to $X$ since

$$
\begin{aligned}
\left\|G\left(t, X_{1}\right)-G\left(t, X_{2}\right)\right\|_{\infty} \leq\left[U \int_{\mathbb{R}} J(m) \mathrm{e}^{T|m|} d m+U+\max _{[0, T]}\left|\bar{m}_{v}\right|\right] & \left\|X_{1}-X_{2}\right\|_{\infty} \\
& \text { for all } t \in[0, T] \text { and } X_{1}, X_{2} \in L^{\infty}(\mathbb{R}) .
\end{aligned}
$$

Therefore, Cauchy-Lipschitz theorem yields the existence and uniqueness of a solution $h \in$ $\mathcal{C}^{1}\left([0, T], L^{\infty}(\mathbb{R})\right)$ of problem $(49)$.

Proof of Lemma 4.3. Let $\tau, \bar{m}, h_{1}$ and $h_{2}$ satisfy the assumptions of the lemma. With a slight abuse of notations, we write $h_{1}(t)=h_{1}(t, \cdot)$ and $h_{2}(t)=h_{2}(t, \cdot)$ for $t \in[0, \tau]$. Set

$$
\mathcal{L}_{t}(h):=U\left(J_{t} \star h-h\right)-\bar{m}(t) h
$$

for $t \in[0, \tau]$ and $h \in L^{\infty}(\mathbb{R})$, and

$$
a(t)=\left(h_{2}^{\prime}(t)-\mathcal{L}_{t}\left(h_{2}(t)\right)\right)-\left(h_{1}^{\prime}(t)-\mathcal{L}_{t}\left(h_{1}(t)\right)\right)
$$

for $t \in[0, \tau]$. Notice that $a \in \mathcal{C}\left([0, \tau], L^{\infty}(\mathbb{R})\right)$, from (6), (7), (20) and Lebesgue's dominated convergence theorem. Now define, for $t \in[0, \tau]$,

$$
w(t)=\left(h_{2}(t)-h_{1}(t)\right) \mathrm{e}^{K t} \in L^{\infty}(\mathbb{R}),
$$

with $K=U+\max _{[0, \tau]}|\bar{m}|$. It is straightforward to check that $w$ is a solution of the ordinary differential equation

$$
w^{\prime}(t)=F(t, w), \quad t \in[0, \tau],
$$

in $L^{\infty}(\mathbb{R})$, for some function $F:[0, \tau] \times L^{\infty}(\mathbb{R}) \rightarrow L^{\infty}(\mathbb{R})$ defined by

$$
F(t, w)=U J_{t} \star w+w(K-U-\bar{m}(t))+a(t) \mathrm{e}^{K t} .
$$

As above, the function $F$ is continuous in $[0, \tau] \times L^{\infty}(\mathbb{R})$ and, as in the proof of Lemma 4.2, it is uniformly Lipschitz-continuous with respect to $w$. We can then define $\widetilde{w} \in \mathcal{C}^{1}\left([0, \tau], L^{\infty}(\mathbb{R})\right)$ as the unique solution of $\widetilde{w}^{\prime}(t)=\max \{F(t, \widetilde{w}(t)), 0\}$ in $[0, \tau]$ with $\widetilde{w}(0)=w(0)$, that is,

$$
\widetilde{w}(t)=w(0)+\int_{0}^{t} \max \{F(s, \widetilde{w}(s)), 0\} d s .
$$

We have $\widetilde{w}(t) \geq w(0)$ and $w(0) \geq 0$ a.e. in $\mathbb{R}$ by assumption. Additionally, from (28), there holds $a(t) \geq 0$ a.e. in $\mathbb{R}$, for all $t \in[0, \tau]$. As a consequence, and since $K-U-\bar{m}(t) \geq 0$ on $[0, \tau]$, one infers that, for all $t \in[0, \tau], F(t, \widetilde{w}(t)) \geq 0$ a.e. in $\mathbb{R}$. We deduce that $\widetilde{w}$ is also a solution of the equation (50) satisfied by $w$. From Cauchy-Lipschitz theorem, we deduce that, for all $t \in[0, \tau], w(t)=\widetilde{w}(t) \geq 0$ and therefore $h_{1}(t, \cdot) \leq h_{2}(t, \cdot)$ a.e. in $\mathbb{R}$. The proof of Lemma 4.3 is thereby complete.

Lemma 4.3 also implies a comparison principle for equations of the form (1). We do not need it explicitly in the proof of Theorem 2.2. However, we believe it to be independent interest and we therefore state it below. 
Lemma 4.6 Let $\tau \in(0,+\infty)$ and $\bar{m} \in C([0, \tau])$. Let $p_{1}, p_{2}$ be such that $p_{i}(t, m) \mathrm{e}^{-t m} \in$ $C^{1}\left([0, \tau], L^{\infty}(\mathbb{R})\right)$ for $i=1,2$, and, for any $t \in[0, \tau]$,

$$
\partial_{t} p_{1}-U\left(J \star p_{1}-p_{1}\right)-(m-\bar{m}(t)) p_{1} \leq \partial_{t} p_{2}-U\left(J \star p_{2}-p_{2}\right)-(m-\bar{m}(t)) p_{2},
$$

a.e. in $m \in \mathbb{R}$. Assume that $p_{1}(0, \cdot) \leq p_{2}(0, \cdot)$ a.e. in $\mathbb{R}$ at initial time. Then, for all $t \in[0, \tau]$, $p_{1}(t, \cdot) \leq p_{2}(t, \cdot)$ a.e. in $\mathbb{R}$.

Proof of Lemma 4.4. Let us first show (33). We consider

$$
\phi(t)=\int_{\mathbb{R}} p(t, m) d m
$$

for $t \in\left[0, T_{\max }\right)$. By using (32) for every $T \in\left[0, T_{\max }\right)$ and integrating (1) over $\mathbb{R}$ with respect to $m$, we obtain that $\phi$ is of class $\mathcal{C}^{1}\left(\left[0, T_{\max }\right)\right)$ and, for every $t \in\left[0, T_{\max }\right)$,

$$
\phi^{\prime}(t)=\int_{\mathbb{R}} \partial_{t} p(t, m) d m=U \int_{\mathbb{R}}(J \star p)(t, m) d m-U \phi(t)+\bar{m}(t)-\bar{m}(t) \phi(t) .
$$

From assumption (6), we have

$$
\int_{\mathbb{R}}(J \star p)(t, m) d m=\int_{\mathbb{R}} p(t, m) d m=\phi(t) .
$$

Finally, $\phi^{\prime}(t)=\bar{m}(t)(1-\phi(t))$ for all $t \in\left[0, T_{\max }\right)$. From assumption (4), there holds $\phi(0)=1$, and since $\bar{m} \in \mathcal{C}\left(\left[0, T_{\max }\right)\right)$, it follows immediately that $\phi(t)=1$ for all $t \in\left[0, T_{\max }\right)$.

Let us now turn to the proof of (34). By integrating (1) between 0 and $t \in\left[0, T_{\max }\right)$, multiplying by $m$, integrating over $\mathbb{R}$ and using (32), we get that, for every $t \in\left[0, T_{\max }\right)$,

$$
\begin{aligned}
\bar{m}(t)-\bar{m}(0) & =\int_{\mathbb{R}} \int_{0}^{t} m[U((J \star p)(s, m)-p(s, m))+(m-\bar{m}(s)) p(s, m)] d s d m \\
& =\int_{0}^{t} \int_{\mathbb{R}} m[U((J \star p)(s, m)-p(s, m))+(m-\bar{m}(s)) p(s, m)] d m d s .
\end{aligned}
$$

Using (6), (33) and Fubini's theorem, one infers that, for all $s \in[0, t]\left(\subset\left[0, T_{\max }\right)\right)$,

$$
\begin{aligned}
\int_{\mathbb{R}} m(J \star p)(s, m) d m & =\int_{\mathbb{R}}\left(\int_{\mathbb{R}}(m-y+y) J(y) p(s, m-y) d y\right) d m \\
& =\bar{m}(s) \int_{\mathbb{R}} J(y) d y+\int_{\mathbb{R}} y J(y) d y=\bar{m}(s)+\mu_{J},
\end{aligned}
$$

with $\mu_{J}=\int_{\mathbb{R}} y J(y) d y$. Then, using the fact that the functions $\bar{m}$ and $t \mapsto \int_{\mathbb{R}} m^{2} p(t, m) d m$ are continuous in $\left[0, T_{\max }\right)$, we deduce that, for every $t \in\left[0, T_{\max }\right)$,

$$
\bar{m}(t)-\bar{m}(0)=t U \mu_{J}+\int_{0}^{t} \int_{\mathbb{R}} m^{2} p(s, m) d m d s-\int_{0}^{t}(\bar{m}(s))^{2} d s .
$$

Then, by Cauchy-Schwarz inequality and (33), we have

$$
(\bar{m}(s))^{2}=\left(\int_{\mathbb{R}} m p(s, m) d m\right)^{2} \leq\left(\int_{\mathbb{R}} p(s, m) d m\right)\left(\int_{\mathbb{R}} m^{2} p(s, m) d m\right)=\int_{\mathbb{R}} m^{2} p(s, m) d m
$$


for all $s \in[0, t]\left(\subset\left[0, T_{\max }\right)\right)$. Therefore,

$$
\bar{m}(t) \geq \bar{m}(0)+t U \mu_{J} \text { for all } t \in\left[0, T_{\max }\right)
$$

and the proof of Lemma 4.4 is thereby complete.

Proof of Lemma 4.5. Since the proof is elementary, we just sketch it. First of all, from (4)(5), the function $C_{0}$ defined in (14) is of class $C^{\infty}([0,+\infty))$ with

$$
C_{0}^{\prime}(t)=\frac{\int_{\mathbb{R}} m p_{0}(m) \mathrm{e}^{t m} d m}{\int_{\mathbb{R}} p_{0}(m) \mathrm{e}^{t m} d m}
$$

and

$$
C_{0}^{\prime \prime}(t)=\frac{\left(\int_{\mathbb{R}} m^{2} p_{0}(m) \mathrm{e}^{t m} d m\right)\left(\int_{\mathbb{R}} p_{0}(m) \mathrm{e}^{t m} d m\right)-\left(\int_{\mathbb{R}} m p_{0}(m) \mathrm{e}^{t m} d m\right)^{2}}{\left(\int_{\mathbb{R}} p_{0}(m) \mathrm{e}^{t m} d m\right)^{2}}
$$

for all $t \geq 0$. As in the proof of Lemma 4.4 above, it follows from Cauchy-Schwarz inequality that $C_{0}^{\prime \prime}(t) \geq 0$ for all $t \geq 0$, that is, $C_{0}$ is convex in $[0,+\infty)$. Hence, the limit of $C_{0}^{\prime}(t)$ as $t \rightarrow$ $+\infty$ exists in $(-\infty,+\infty]$. To show that this limit is equal to $m_{0}$, let us first consider the case $m_{0}=+\infty$. In that case, for any $0<M<M^{\prime}<+\infty$ and $t \geq 0$, one has $\int_{-\infty}^{M} p_{0}(m) \mathrm{e}^{t m} d m \leq$ $\mathrm{e}^{t M}$ and $\int_{M}^{+\infty} p_{0}(m) \mathrm{e}^{t m} d m \geq \mathrm{e}^{t M^{\prime}} \int_{M^{\prime}}^{+\infty} p_{0}(m) d m$, and since $\int_{M^{\prime}}^{+\infty} p_{0}(m) d m>0$, one gets that

$$
\int_{\mathbb{R}} p_{0}(m) \mathrm{e}^{t m} d m \sim \int_{M}^{+\infty} p_{0}(m) \mathrm{e}^{t m} d m \text { as } t \rightarrow+\infty
$$

Furthermore, $\left|\int_{-\infty}^{M} m p_{0}(m) \mathrm{e}^{t m} d m\right|=O\left(\mathrm{e}^{t M}\right)$ as $t \rightarrow+\infty$, while $\int_{M}^{+\infty} m p_{0}(m) \mathrm{e}^{t m} d m \geq$ $M^{\prime} \mathrm{e}^{t M^{\prime}} \int_{M^{\prime}}^{+\infty} p_{0}(m) d m$. Hence, $\int_{\mathbb{R}} m p_{0}(m) \mathrm{e}^{t m} d m \sim \int_{M}^{+\infty} m p_{0}(m) \mathrm{e}^{t m} d m$ as $t \rightarrow+\infty$. From (51) and the previous calculations, it follows that, for every $M>0$,

$$
\lim _{t \rightarrow+\infty} C_{0}^{\prime}(t)=\lim _{t \rightarrow+\infty} \frac{\int_{M}^{+\infty} m p_{0}(m) \mathrm{e}^{t m} d m}{\int_{M}^{+\infty} p_{0}(m) \mathrm{e}^{t m} d m} \geq M
$$

and, since $M$ can be arbitrarily large, one concludes that $\lim _{t \rightarrow+\infty} C_{0}^{\prime}(t)=+\infty$.

When $m_{0}$ is finite, then

$$
C_{0}^{\prime}(t)=m_{0}+\frac{\int_{-\infty}^{0} m p_{0}\left(m_{0}+m\right) \mathrm{e}^{t m} d m}{\int_{-\infty}^{0} p_{0}\left(m_{0}+m\right) \mathrm{e}^{t m} d m} .
$$

Hence, even if it means working with $q_{0}(m):=p_{0}\left(m_{0}+m\right)$, one can assume without loss of generality that $m_{0}=0$. As above, it is easy to check that, for any $M<0, \int_{-\infty}^{0} p_{0}(m) \mathrm{e}^{t m} d m \sim$ 
$\int_{M}^{0} p_{0}(m) \mathrm{e}^{t m} d m$ as $t \rightarrow+\infty$. Furthermore, for any $M<M^{\prime}<0, \int_{-\infty}^{M} m p_{0}(m) \mathrm{e}^{t m} d m=$ $O\left(\mathrm{e}^{t M}\right)$ as $t \rightarrow+\infty$, while

$$
\int_{M}^{0} m p_{0}(m) \mathrm{e}^{t m} d m \leq \int_{M^{\prime}}^{0} m p_{0}(m) \mathrm{e}^{t m} d m \leq \mathrm{e}^{t M^{\prime}} \int_{M^{\prime}}^{0} m p_{0}(m) d m
$$

and $\int_{M^{\prime}}^{0} m p_{0}(m) d m<0$ by definition of $m_{0}$ (which is equal to 0 in this argument). Hence, $\int_{-\infty}^{0} m p_{0}(m) \mathrm{e}^{t m} d m \sim \int_{M}^{0} m p_{0}(m) \mathrm{e}^{t m} d m$ as $t \rightarrow+\infty$. Therefore, for every $M<0$,

$$
\lim _{t \rightarrow+\infty} C_{0}^{\prime}(t)=\lim _{t \rightarrow+\infty} \frac{\int_{M}^{0} m p_{0}(m) \mathrm{e}^{t m} d m}{\int_{M}^{0} p_{0}(m) \mathrm{e}^{t m} d m} .
$$

Since the absolute value of the right-hand side is bounded by $|M|$ and since $M$ can be chosen arbitrarily close to 0 , one concludes that $\lim _{t \rightarrow+\infty} C_{0}^{\prime}(t)=0$.

Let us now assume that $m_{0}<+\infty$ and prove (43), that is, $\lim _{t \rightarrow+\infty} C_{0}^{\prime \prime}(t)=0$. First of all, (51) and (52) imply that, for every $t \geq 0$,

$$
C_{0}^{\prime \prime}(t)=-m_{0}^{2}+2 m_{0} C_{0}^{\prime}(t)-C_{0}^{\prime}(t)^{2}+\underbrace{\frac{\int_{-\infty}^{0} m^{2} p_{0}\left(m_{0}+m\right) \mathrm{e}^{t m} d m}{\int_{-\infty}^{0} p_{0}\left(m_{0}+m\right) \mathrm{e}^{t m} d m}}_{=: f(t)} .
$$

Therefore, since $\lim _{t \rightarrow+\infty} C_{0}^{\prime}(t)=m_{0}$, one only has to show that $f(t) \rightarrow 0$ as $t \rightarrow+\infty$. Even if it means working with $q_{0}(m):=p_{0}\left(m_{0}+m\right)$, one can assume without loss of generality that $m_{0}=0$ in the definition of $f(t)$. Observe that $f(t) \geq 0$ for all $t \geq 0$. Furthermore, as it was done in the previous paragraph, one can easily check that, for any $M<0$,

$$
\limsup _{t \rightarrow+\infty} f(t)=\limsup _{t \rightarrow+\infty} \frac{\int_{M}^{0} m^{2} p_{0}(m) \mathrm{e}^{t m} d m}{\int_{M}^{0} p_{0}(m) \mathrm{e}^{t m} d m} .
$$

Since the right-hand side ranges between 0 and $M^{2}$ and $M$ can be chosen arbitrarily close to 0 , one finally concludes that $f(t) \rightarrow 0$ as $t \rightarrow+\infty$. Hence, (43) holds and the proof of Lemma 4.5 is thereby complete.

\section{References}

Alfaro, M. and R. Carles (2014). Explicit solutions for replicator-mutator equations: Extinction versus acceleration. SIAM Journal on Applied Mathematics 74(6), 1919-1934.

Alfaro, M. and J. Coville (2016). Propagation phenomena in monostable integro-differential equations: acceleration or not? arXiv preprint arXiv:1610.05908. 
Alfaro, M., J. Coville, and G. Raoul (2014). Bistable travelling waves for nonlocal reaction diffusion equations. Disc Cont Dyn Systems A 34, 1775-1791.

Berestycki, H., G. Nadin, B. Perthame, and L. Ryzhik (2009). The non-local Fisher-KPP equation: travelling waves and steady states. Nonlinearity 22(12), 2813-2844.

Carr, A. and J. Chmaj (2004). Uniqueness of travelling waves for nonlocal monostable equations. Proc. Amer. Math. Soc. 132(8), 2433-2439.

Couce, A. and O. A. Tenaillon (2015). The rule of declining adaptability in microbial evolution experiments. Frontiers in genetics 6, 99 .

Coville, J. and L. Dupaigne (2007). On a nonlocal reaction diffusion equation arising in population dynamics. Proc $R$ Soc Edin A 137, 1-29.

Desai, M. M. and D. S. Fisher (2011). The balance between mutators and nonmutators in asexual populations. Genetics 188(4), 997-1014.

Elena, S. F. and R. E. Lenski (2003). Evolution experiments with microorganisms: the dynamics and genetic bases of adaptation. Nature Reviews Genetics 4(6), 457-469.

Fang, J. and X.-Q. Zhao (2011). Monotone wavefronts of the nonlocal Fisher-KPP equation. Nonlinearity 24, 3043-3054.

Faye, G. and M. Holzer (2015). Modulated traveling fronts for a nonlocal Fisher-KPP equation: a dynamical systems approach. J. Diff. Equations 258(7), 2257-2289.

Garnier, J. (2011). Accelerating solutions in integro-differential equations. SIAM J Math Anal 43, 1955-1974.

Garnier, J., F. Hamel, and L. Roques (2016). Transition fronts and stretching phenomena for a general class of reaction-dispersion equations. Disc Cont Dyn Systems A in press.

Genieys, S., V. Volpert, and V. Auger (2006). Pattern and waves for a model in population dynamics with nonlocal consumption of resources. Math. Model. Nat. Phenom. 1, 63-80.

Gerrish, P. J., A. Colato, A. S. Perelson, and P. D. Sniegowski (2007). Complete genetic linkage can subvert natural selection. Proceedings of the National Academy of Sciences 104(15), 6266-6271.

Gerrish, P. J. and R. E. Lenski (1998). The fate of competing beneficial mutations in an asexual population. Genetica 102, 127-144.

Good, B. H. and M. M. Desai (2013). Fluctuations in fitness distributions and the effects of weak linked selection on sequence evolution. Theoretical population biology 85, 86-102.

Good, B. H. and M. M. Desai (2014). Deleterious passengers in adapting populations. Genetics 198(3), 1183-1208.

Good, B. H., I. M. Rouzine, D. J. Balick, O. Hallatschek, and M. M. Desai (2012). Distribution of fixed beneficial mutations and the rate of adaptation in asexual populations. Proceedings of the National Academy of Sciences 109(13), 4950-4955. 
Gourley, S. A. (2000). Travelling front solutions of a nonlocal fisher equation. J. Math. Biol. 41, $272-284$.

Hamel, F. and L. Ryzhik (2014). On the nonlocal fisher-kpp equation: steady states, spreading speed and global bounds. Nonlinearity $27(11), 2735$.

Martin, G. and L. Roques (2016). The non-stationary dynamics of fitness distributions: Asexual model with epistasis and standing variation. Genetics, DOI: 10.1534/genetics.116.187385.

Martin-Löf, A. (1973). Mixing properties, differentiability of the free energy and the central limit theorem for a pure phase in the ising model at low temperature. Communications in Mathematical Physics 32(1), 75-92.

Park, S.-C., D. Simon, and J. Krug (2010). The speed of evolution in large asexual populations. Journal of Statistical Physics 138(1-3), 381-410.

Schumacher, K. (1980). Travelling-front solutions for integro-differential equations. I. J. Reine Angew. Math. 316, 54-70.

Sniegowski, P. D. and P. J. Gerrish (2010). Beneficial mutations and the dynamics of adaptation in asexual populations. Philosophical Transactions of the Royal Society B: Biological Sciences 365(1544), 1255-1263.

Tsimring, L. S., H. Levine, and D. A. Kessler (1996). Rna virus evolution via a fitness-space model. Physical review letters 76(23), 4440.

Weinberger, H. F. (1982). Long-time behavior of a class of biological models. SIAM J Math Anal 13(3), 353-396.

Weinberger, H. F. (2002). On spreading speeds and traveling waves for growth and migration in periodic habitat. J Math Biol 45, 511-548.

Yagisita, H. (2009). Existence and nonexistence of travelling waves for a nonlocal monostable equation. Publ. RIMS, Kyoto Univ. 45, 925-953. 